\title{
A Time-Dependent Neutron Transport Method of Characteristics Formulation with Time Derivative Propagation
}

\author{
Adam J. Hoffman and John C. Lee \\ adamhoff@umich.edu, jcl@umich.edu \\ University of Michigan, College of Engineering \\ Department of Nuclear Engineering and Radiological Sciences \\ 2355 Bonisteel Boulevard, Ann Arbor, Michigan 48109-2104
}

\begin{abstract}
A new time-dependent Method of Characteristics (MOC) formulation for nuclear reactor kinetics was developed utilizing angular flux time-derivative propagation. This method avoids the requirement of storing the angular flux at previous points in time to represent a discretized time derivative; instead, an equation for the angular flux time derivative along 1D spatial characteristics is derived and solved concurrently with the 1D transport characteristic equation. This approach allows the angular flux time derivative to be recast principally in terms of the neutron source time derivatives, which are approximated to high-order accuracy using the backward differentiation formula (BDF). This approach, called Source Derivative Propagation (SDP), drastically reduces the memory requirements of time-dependent MOC relative to methods that require storing the angular flux. An SDP method was developed for 2D and 3D applications and implemented in the computer code DeCART in 2D. DeCART was used to model two reactor transient benchmarks: a modified TWIGL problem and a C5G7 transient. The SDP method accurately and efficiently replicated the solution of the conventional time-dependent MOC method using two orders of magnitude less memory.
\end{abstract}

Keywords: Method of Characteristics (MOC), Reactor Kinetics, Backward Differentiation Formula (BDF) 


\section{Introduction}

We are in the midst of a transition in the state-of-the art for reactor kinetics. Until recently, reactor transients have been principally modeled using the diffusion approximation to the transport equation [1]. While diffusion methods are computationally efficient, there is growing interest in reactor designs and fuels for which diffusion may not be sufficiently accurate. Consequently, there is interest in the direct use of neutron transport methods for nuclear reactor kinetics [2]-[8], and this is increasingly practical due to the steady advances in computational power.

Method of Characteristics (MOC) [9],[10] is a popular method for solving the steady-state neutron transport equation, especially for small, assembly-level models. Its popularity stems from its ability to resolve complex geometry without spatial homogenization. As with other transport methods, it has been extended to solve the time-dependent transport equation as well.

Most reactor kinetics methods in neutron transport and diffusion treat time dependence by discretizing the equations in time and applying a conventional time integration method to approximate the time derivative (e.g. Backward Euler, Theta Method, Runge-Kutta, etc.). In this case, the spatial and angular dependence are treated using the same approach that would be employed to solve the steadystate transport equation (e.g. PN, SN, MOC, etc.). The result is that the time-dependent transport problem is reduced to a series of pseudo-steady-state problems at discrete points in time coupled to previous time points through the update of state variables and the treatment of the time derivatives. This approach is used in many time-dependent neutron transport codes, including the threedimensional whole core neutron transport code DeCART [4],[11],[12] which was used in this work. 
One obstacle with this approach is that it implies that the neutron angular flux ${ }^{1}$ should be stored from one or more previous time steps in order to represent the angular flux time derivative. Although the angular flux is the fundamental solution of the transport equation, the neutron scalar flux ${ }^{2}$ is often the desired solution in practice. The scalar flux is the integral of the angular flux over all angles in space, and it is generally used to calculate reaction rates. Thus in steady-state transport the angular flux is not generally stored; it is instead numerically integrated as it is generated to calculate the scalar flux. Storing the angular flux for large reactor models requires staggering amounts of memory that can exceed the capabilities of even leading-class supercomputers. As a result, time-dependent neutron transport codes are either very limited in the size or resolution of the reactor transients they can model, or they employ low-order angular or spatial approximations to treat the angular flux time derivative.

To circumvent the angular flux storage issue, we investigated an alternative class of timedependent transport methods called time-derivative transport, whereby a transport equation is derived for the angular flux time derivative and simultaneously solved with the transport equation for the angular flux. When this concept is applied to MOC, the angular flux time derivative is solved by propagation in terms of the neutron source ${ }^{3}$ time derivatives. As a result, we call this method Source Derivative Propagation (SDP).

One significant advantage of SDP is that it can be inexpensively extended to high-order accuracy by approximating the source time derivatives using the Backward Differentiation Formula (BDF). By contrast, if BDF is applied directly to the angular flux time derivative for time-dependent MOC, the memory problem is significantly exacerbated. For the problems examined in this work, SDP method with

\footnotetext{
${ }^{1}$ The neutron angular flux is the solution of the neutron transport equation and is defined as the instantaneous neutron path rate density in space, angle, and energy.

${ }^{2}$ The neutron scalar flux is the neutron angular flux integrated over all directions. The scalar flux is of interest to nuclear reactor engineers because it is used to calculate nuclear reaction rates.

${ }^{3}$ In many neutron transport methods, the neutron source terms (e.g. fission, decay, etc.) are combined into a single term which is updated iteratively.
} 
high-order BDF approximations to the source derivatives accurately replicated the conventional, highorder time-dependent MOC solution when the same BDF order and time steps were used.

Other researchers have also recently investigated angular flux time derivative approximations for time-dependent MOC. Talamo developed three conventional time-dependent MOC formulations which represent the current practice for time-dependent MOC [7]. Talamo's Method II stored the angular flux to represent the time derivative while Methods I \& III approximated the time derivative using the scalar flux. Method II is equivalent to our reference method for assessing the accuracy of SDP while Method I is equivalent to our reference method for assessing computational efficiency. While Talamo observed that the methods that approximated the time derivative as isotropic were generally accurate, Method I was unstable with very short time steps. This instability was not observed in this work because we did not require such small time steps.

In contrast to Talamo, Tsujita addressed the angular flux memory issue by developing a timedependent $\mathrm{MOC}$ formulation that recalculated the previous angular fluxes on the fly [8]. While in principle this could be done for an arbitrary number of time steps, with each successive time step the method becomes linearly more computationally expensive. To limit this expense, Tsujita only recalculated the angular fluxes for a few previous time steps and used an isotropic approximation for the earliest recalculated time step. While the isotropic approximation introduces some error, the error is mitigated because with each earlier time step recalculated on the fly, the error term is multiplied by an additional inverse-velocity term, which is very small. While the derivation of Tsujita's on-the-fly (OTF) method is very different from the SDP method explored in this work, it has similar computational and memory requirements. The primary advantage of SDP over OTF is that SDP is more inexpensively extended to high-order accuracy in time using BDF.

A high fidelity alternative to applying a finite differencing technique to treat the angular flux time derivative for time-dependent $\mathrm{MOC}$ is to map the characteristics to the time domain as well as the 
spatial domain. This space-time transport is in principle very accurate because it explicitly accounts for neutron time of flight, but it is substantially more computationally expensive and memory intensive than methods like SDP and OTF. Pandya and Adams developed a 3D space-time MOC method for radiative transfer using this approach [13], while Tsujita et al. implemented a 1D space-time MOC method for reactor kinetics [14]. We originally developed the early SDP methods using a space-time characteristic formulation with coarse approximations in the time domain [15]; the derivation presented in this work is equivalent but more succinct.

In Section 2 we will provide the time-dependent MOC formulations used in this work, including SDP. Section 2.1 will briefly present two conventional time-dependent MOC methods which are representative of the state of the art. In Section 2.2 we will derive the primary SDP method used in this work. Other SDP methods were evaluated in [16]. In Section 3 we will present two reactor transient benchmark problems: a comparatively simple TWIGL problem[17] and a more challenging C5G7 transient[18]. After presenting each benchmark problem we will assess the accuracy and efficiency of the SDP method by comparison to the reference methods. A summary and conclusions are presented in Section 4.

\section{Time-Dependent MOC Formulations}

MOC has been used extensively to solve the steady-state neutron transport equation for nuclear reactor physics, especially for two-dimensional (2D) assembly-level problems. In the last decade, MOC has also been applied to the time-dependent, multigroup neutron transport equation:

$$
\frac{1}{v_{g}} \frac{d \varphi_{g}(\mathbf{x}, \mathbf{\Omega}, t)}{d t}=-\mathbf{\Omega} \cdot \nabla \varphi_{g}(\mathbf{x}, \mathbf{\Omega}, t)-\Sigma_{t, g}(\mathbf{x}, t) \varphi_{g}(\mathbf{x}, \mathbf{\Omega}, t)+q_{g}(\mathbf{x}, \mathbf{\Omega}, t)
$$

where

$$
\begin{aligned}
& v_{g}=\text { neutron velocity }\left[\mathrm{cm} \cdot \mathrm{s}^{-1}\right] \\
& g=\text { neutron kinetic energy group index }
\end{aligned}
$$




$$
\begin{aligned}
& \varphi_{g}=\text { neutron angular flux }\left[\mathrm{cm}^{-2} \cdot \mathrm{s}^{-1}\right] \\
& \mathbf{x}=\text { position vector }=x \mathbf{i}+y \mathbf{j}+z \mathbf{k} \\
& \mathbf{\Omega}=\text { unit direction of flight vector }=\sqrt{1-\mu^{2}} \cos \alpha \mathbf{i}+\sqrt{1-\mu^{2}} \sin \alpha \mathbf{j}+\mu \mathbf{k} \\
& \alpha=\text { azimuthal angle } \\
& \mu=\cos \theta=\text { cosine of the polar angle } \theta \\
& t=\text { time }[\mathrm{s}] \\
& \Sigma_{t, g}=\text { macroscopic total } \mathrm{cross} \mathrm{section}^{4}\left[\mathrm{~cm}^{-1}\right] \\
& q_{g}=\text { total neutron source }\left[\mathrm{cm}^{-3} \cdot \mathrm{s}^{-1}\right] .
\end{aligned}
$$

The total neutron source includes fission, scattering, and delayed neutrons:

$$
\begin{aligned}
q_{g}(\mathbf{x}, \mathbf{\Omega}, t)= & (1-\beta) \frac{\chi_{p, g}(\mathbf{x})}{4 \pi} \sum_{g^{\prime}}^{G} \int_{4 \pi} v \Sigma_{f, g^{g}}(\mathbf{x}, t) \varphi_{g^{\prime}}\left(\mathbf{x}, \mathbf{\Omega}^{\prime}, t\right) d \mathbf{\Omega}^{\prime} \\
& +\sum_{g^{\prime}}^{G} \int_{4 \pi} \Sigma_{s, g^{\prime} \rightarrow g}\left(\mathbf{x}, \mathbf{\Omega}^{\prime} \cdot \mathbf{\Omega}, t\right) \varphi_{g^{\prime}}\left(\mathbf{x}, \mathbf{\Omega}^{\prime}, t\right) d \mathbf{\Omega}^{\prime}+\frac{S_{d, g}(\mathbf{x}, t)}{4 \pi},
\end{aligned}
$$

where

$$
\begin{aligned}
& \beta=\text { total delayed neutron fraction } \\
& \chi_{p, g}=\text { prompt neutron fission spectrum } \\
& v=\text { number of neutrons released per fission } \\
& \Sigma_{f, g}=\text { macroscopic fission cross section }\left[\mathrm{cm}^{-1}\right] \\
& \Sigma_{g}\left(\mathbf{x}, \boldsymbol{\Omega}^{\prime} \cdot \Omega, E^{\prime} \rightarrow E\right)=\text { macroscopic differential scattering cross section }\left[\mathrm{cm}^{-1} \cdot \mathrm{MeV}^{-1}\right] .
\end{aligned}
$$

The delayed neutron source $S_{d, g}$ is the total contribution of the delayed neutron precursors:

\footnotetext{
${ }^{4}$ The macroscopic total cross section is the probability per unit length traveled that a neutron will undergo any nuclear reaction. Similarly, other macroscopic cross sections are defined for specific nuclear reactions (e.g. fission, scattering, radiative capture, etc.). For the purpose of the benchmark problems investigated in this work, these are known quantities.
} 


$$
S_{d, g}(\mathbf{x}, t)=\sum_{j}^{J} \chi_{d_{, g} j}(\mathbf{x}) \lambda_{j} C_{j}(\mathbf{x}, t)
$$

where $j$ is the index for each delayed neutron precursor group, and where the delayed neutron

precursor concentration is described by the differential equation:

$$
\frac{d C_{j}(\mathbf{x}, t)}{d t}=\sum_{g^{\prime}}^{G} \int_{4 \pi} \beta_{j} v \Sigma_{f, g^{\prime}}(\mathbf{x}, t) \varphi_{g^{\prime}}\left(\mathbf{x}, \mathbf{\Omega}^{\prime}, t\right) d \mathbf{\Omega}^{\prime}-\lambda_{j} C_{j}(\mathbf{x}, t),
$$

where

$$
\begin{aligned}
& \chi_{d, j}=\text { delayed neutron spectrum for precursor group } j \\
& \lambda_{j}=\text { decay constant for delayed neutron precursor group } j\left[\mathrm{~s}^{-1}\right] \\
& C_{j}=\text { delayed neutron precursor concentration for precursor group } j\left[\mathrm{~cm}^{-3}\right] \\
& \beta_{j}=\text { delayed neutron precursor yield for precursor group } j .
\end{aligned}
$$

The basic approach of MOC is to convert the 3D transport equation into a family of $1 \mathrm{D}$ transport equations spanning the spatial and angular domain. With some approximations, the $1 \mathrm{D}$ equations can be solved inexpensively, and the results can be recombined to generate the solution to the 3D transport equation. Using our nomenclature, the 3D transport equation is rewritten along an arbitrary characteristic with the index $k$ and the spatial dimension $s$ from an arbitrary position $\mathbf{x}_{0}$ in the direction $\boldsymbol{\Omega}_{l, m}$ defined by azimuthal angle $\alpha_{l}$ and polar angle $\theta_{m}$.

The MOC equation along the characteristic can be solved analytically with some approximations. First we use transport-corrected scattering ${ }^{5}$ to approximate the neutron source as isotropic:

\footnotetext{
${ }^{5}$ Transport-corrected scattering and the flat source approximation are common in MOC for reactor kinetics. The transient methods described in this work are compatible with more rigorous treatment of these parameters (e.g. anisotropic scattering and spatially-variant sources), though it increases the complexity of the equations.
} 


$$
\frac{1}{v_{g}} \frac{d \varphi_{g}^{k}(s, t)}{d t}=-\frac{d \varphi_{g}^{k}(s, t)}{d s}-\Sigma_{t r, g}^{k}(s, t) \varphi_{g}^{k}(s, t)+q_{g}^{k}(s, t)
$$

where the isotropic neutron source is:

$$
q_{g}^{k}(s, t)=\frac{1}{4 \pi}\left[\sum_{g^{\prime}=1}^{G}\left((1-\beta) \chi_{p, g}(s) v \Sigma_{f, g^{g}}(s, t) \phi_{g^{g}}(s, t)+\Sigma_{s, g^{t} \rightarrow g}^{t r}(s, t) \phi_{g^{g}}(s, t)\right)+S_{d, g}(t)\right.
$$

Next we discretize the geometry into fine spatial regions, and we define the portion of a characteristic within a region as a segment. Figure 1 shows an example meshing for a single fuel rod with arbitrarily-shaped regions and many parallel characteristics at angle $\boldsymbol{\Omega}$. Seven segments are numerated in the bottom right corner.

Within the regions we assume that there is no spatial dependence of the macroscopic cross sections and the neutron source. In addition, we evaluate the equation at time $t_{n}$ using the index $\mathrm{n}$ for time steps. With these approximation, the 1D neutron transport equation is rewritten as:

$$
\frac{1}{v_{g}} \frac{d \varphi_{g}^{k_{g} n}(s)}{d t}=-\frac{d \varphi_{g}^{k, n}(s)}{d s}-\Sigma_{t r, g}^{r, n} \varphi_{g}^{k, n}(s)+q_{g}^{r, n}
$$

where $r$ is the index for the fine spatial region that corresponds to the position $s$ along characteristic $k$.

Equation (7) differs from steady-state MOC the equation due to the presence of the time derivative and the explicit treatment of delayed neutrons. We can partially solve the differential equation along each characteristic by applying the integrating factor $e^{\Sigma_{t r g}^{r n g}\left(s-s_{0}^{k, r}\right)}$ where $s_{0}^{k, r}$ is the incoming position of the characteristic $k$ within region $r$ along $s$. The partial solution of the differential equation is: 


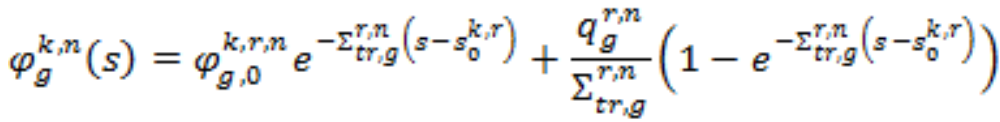

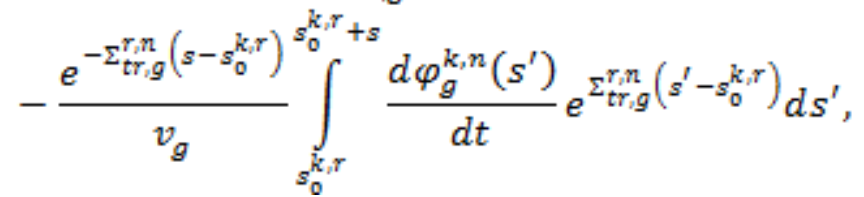

where the incoming angular flux at the beginning of the segment is $\varphi_{g, 0}^{k, r, n} \equiv \varphi_{g}^{k, n}\left(s_{0}^{k, r}\right)$. The incoming angular flux is assumed to be known because the MOC equations are used in a sweeping fashion, evaluating segments sequentially along each characteristic. For simplicity, for the remainder of the equations we will define $s_{0}^{k, r} \equiv 0$ for each segment to enhance readability.

Note that if the angular flux time derivative is zero, the resulting equation is equivalent to steady-state MOC. In steady-state MOC, this equation is used both to propagate the angular flux along characteristics as well as to calculate the average angular flux for the segment. Briefly, the basic approach for steady-state MOC is to evaluate Eq. (8) at the end of each segment to propagate the angular flux. In addition, the segment-wise average angular flux is calculated as an intermediate step to calculate the region-wise scalar fluxes. To avoid the memory expense of storing the angular flux for each segment, the segment-wise average angular fluxes are numerically integrated on the fly to calculate the region-wise average scalar fluxes. After completing an iteration of $\mathrm{MOC}$, if the scalar fluxes have changed substantially from the previous iteration, they are used to update the region-wise neutron source, and another iteration is performed. In steady-state, this process is used to solve the eigenvalue problem or fixed source problem, while for transients it is used to determine the change in the neutron flux as a result of some perturbation. DeCART's neutron transport methods are detailed in [4],[11],[12].

The time-dependent MOC methods used in this work differ in their resolution of the angular flux time derivative term in Eq. (8). Section 2.1 briefly summarizes the conventional approach of approximating the time derivative using a finite difference approximation. Section 2.2 provides the derivation for the new method developed in this work, SDP. 


\subsection{Finite Differenced Time-Dependent MOC}

The typical approach to solving the time-dependent neutron transport equation is to discretize the equation in time and apply a time integration technique like Backward Euler to treat the time derivative. Although there are many time integration methods available, they generally result in one or more equations of similar form to the steady-state transport equation but with a transient fixed source term resulting from a finite difference approximation of the time derivative. In this work we used the Backward Differentiation Formula (BDF) to treat the time derivatives.

\section{Backward Differentiation Formula (BDF)}

Although Backward Euler is the most commonly used time integration technique for transportbased nuclear reactor kinetics, for this work we used the Backward Differentiation Formula (BDF). BDF is an implicit, variable-order time integration technique for solving stiff differential equations. BDF achieves a high-order approximation of time derivatives by utilizing the solution data from previous points in time; a first-order accurate approximation requires one previous data point, a second-order accurate approximation requires two previous data points, and so on. BDF approximations beyond order six are unstable, and the first-order approximation is equivalent to Backward Euler. High-order BDF methods have been successfully used for some reactor kinetics codes based on diffusion theory [19][21], but they have not been applied to any transport-based kinetics codes outside of this work.

Using BDF the time derivative is approximated to order $O_{B D F}$ with:

$$
\frac{d \varphi_{g}^{k_{2} n}\left(s^{\prime}\right)}{d t} \approx \sum_{i=0}^{O_{B D F}} A^{i} \varphi_{g}^{k, n-i}\left(s^{\prime}\right)
$$

where the coefficients $A^{\tilde{i}}$ are a function of the previous time steps [21]: 


$$
A^{i} \equiv\left\{\begin{array}{cc}
\sum_{l=1}^{o_{B D F}} \frac{1}{\sum_{j=0}^{l-1} \Delta t_{n-j}} & i=0 \\
\frac{(-1)^{i} \prod_{l=1_{x} \neq i}^{o_{B D F}} \sum_{j=0}^{l-1} \Delta t_{n-j}}{\prod_{l=1}^{i}\left(\sum_{j=l-1}^{i-1} \Delta t_{n-j}\right) \prod_{l=i+1}^{o_{B D F}}\left(\sum_{j=i}^{l-1} \Delta t_{n-j}\right)} & i \neq 0
\end{array} .\right.
$$

Here the previous time steps are defined by $\Delta t_{n-i} \equiv t_{n-i}-t_{n-i-1}$. Coefficients for constant time steps are widely published in numerical method textbooks. Note that high-order methods are not possible for the initial time steps due to the lack of sufficient previous data; in this implementation when a high-order method is specified by the user and there is insufficient data for that order, the highest order approximation possible is used instead: BDF1 for the first time step, BDF2 for the second time step, etc.

When BDF is applied to the angular flux time derivative in Eq. (8), the equation is rewritten as:

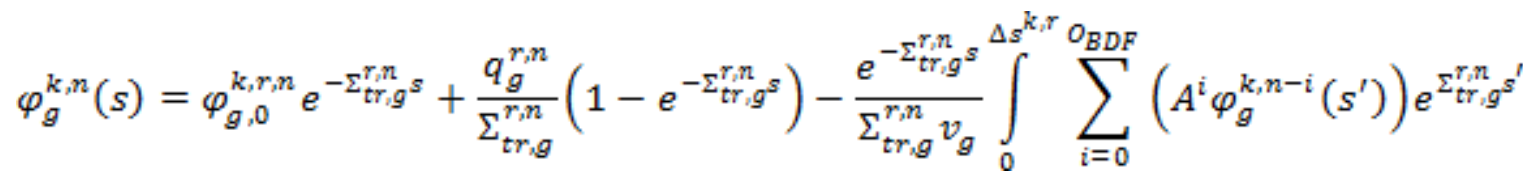

where $O_{B D F}$ is the order of the BDF method, and as a reminder $s_{0}^{k, r} \equiv 0$.

Since the spatial dependence of the angular flux along the segment is unknown, a further approximation is necessary to evaluate the integral. In this work we considered two common approximations to the angular flux for treating the time derivative; the first method serves as a benchmark for accuracy while the second method is a benchmark for computational efficiency.

\subsubsection{Reference BDF MOC (RBDC)}

In the first method (RBDC) we approximate the segment-wise spatially-dependent angular fluxes used for the time derivative in Eq. (11) with the segment-wise average angular fluxes:

$$
\varphi_{g}^{k, n-i}\left(s^{\prime}\right) \approx \bar{\varphi}_{g}^{k, n-i} \equiv \frac{1}{\Delta s^{k, r}} \int_{0}^{\Delta s^{k_{k}, r}} \varphi_{g}^{k_{g}, n-i}\left(s^{\prime}\right) d s^{\prime}
$$


With this approximation we evaluate the remaining integral in Eq. (11):

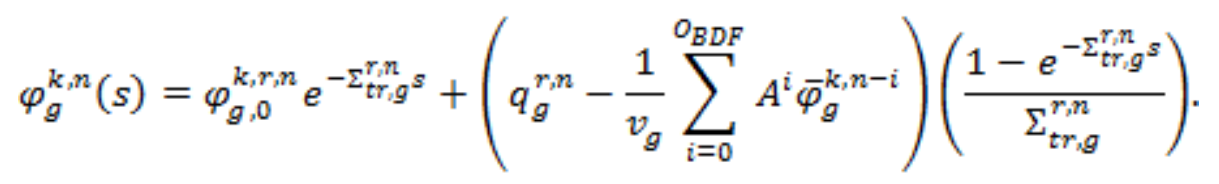

Eq. (13) gives the spatially-dependent segment-wise angular flux $\varphi(s)$ in terms of the segment-

wise average angular flux $\bar{\varphi}$. The average angular flux is calculated by integrating Eq. (13) over the segment and dividing by the segment length:

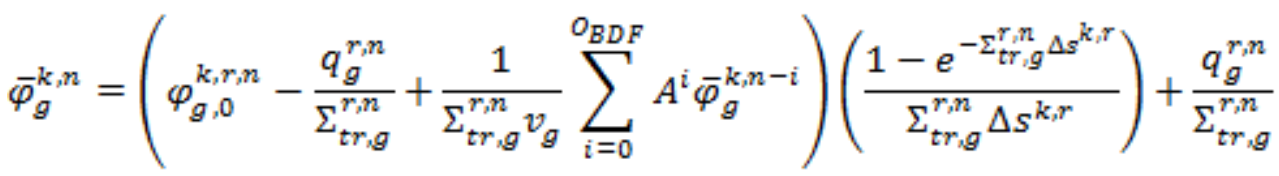

$$
\begin{aligned}
& -\frac{1}{\Sigma_{t r, g}^{r, n} v_{g}} \sum_{i=0}^{O_{B D F}} A^{i} \bar{\varphi}_{g}^{k, n-i}
\end{aligned}
$$

In Eq. (14) the segment-wise average angular flux for the current time step appears on both the LHS and RHS. This dependency is eliminated algebraically:

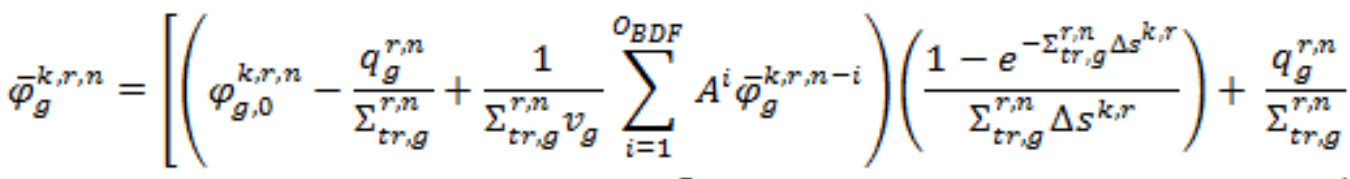

$$
\begin{aligned}
& \left.-\frac{1}{\Sigma_{t r, g}^{r, n} v_{g}} \sum_{i=1}^{O_{B D F}} A^{i} \bar{\varphi}_{g}^{k, r, n-i}\right]\left[1+\frac{A^{0}}{\Sigma_{t r, g}^{r, n} v_{g}}\left(1-\frac{1-e^{-\Sigma_{t r, g}^{r n} \Delta s^{k_{2} r}}}{\Sigma_{t r, g}^{r, n} \Delta s^{k, r}}\right)\right]^{-1} .
\end{aligned}
$$

Equations (13) and (15) are the solutions to the MOC equations for RBDC; Eq. (13) is used to propagate the angular flux along characteristics while Eq. (15) is integrated over space and angle within regions to calculate the region-wise scalar flux. For $\mathrm{MOC}$ - both in steady-state and time-dependent algorithms - the angular flux is evaluated segment by segment in a sweeping fashion along characteristics that span the spatial and angular domain such that region-wise scalar flux is fully integrated after every characteristic has been evaluated. The region-wise scalar flux is used to update the neutron source, and the process repeats iteratively until the source converges. RBDC is a 
straightforward application of BDF to the MOC equations, but storing the segment-wise average angular flux is memory-prohibitive for large reactor models. RBDC with BDF1 (i.e. RBDC1) is equivalent to Talamo's Method II [7].

\subsubsection{Isotropic Approximation BDF MOC (IBDC)}

To avoid a drastic increase in memory requirements over the steady-state method, many neutron transport codes for reactor kinetics approximate the angular dependence of the angular flux time derivative. A popular approximation is to assume that the angular flux time derivative is isotropic and can be approximated using the region-wise scalar flux ${ }^{6}$ :

$$
\sum_{i=0}^{o_{B D F}} A^{i} \bar{\varphi}_{g}^{k_{3} r_{2} n-i} \approx \sum_{i=0}^{o_{B D F}} \frac{A^{i} \phi_{g}^{r, n-i}}{4 \pi}
$$

It is important to note that, with this approximation, the solution will no longer converge to the transport solution as the time steps are refined. We can evaluate the remaining integral in Eq. (11):

$$
\varphi_{g}^{k, n}(s)=\varphi_{g, 0}^{k_{2} r, n} e^{-\Sigma_{t r_{i}, g^{s}}^{r n n}}+\left(q_{g}^{r, n}-\frac{1}{v_{g}} \sum_{i=0}^{o_{B D F}} \frac{A^{i} \phi_{g}^{r, n-i}}{4 \pi}\right)\left(\frac{1-e^{-\Sigma_{t r, g^{s}}^{r n}}}{\Sigma_{t r_{2}, g}^{r, n}}\right)
$$

Equation (17) is the characteristic equation used to propagate the angular flux along the characteristic for IBDC. As for RBDC, the segment-wise angular flux from Eq. (17) is integrated over space and angle within the region to calculate the region-wise scalar flux.

The primary advantage of IBDC over RBDC is that it drastically reduces the memory requirements of time-dependent neutron transport, but a significant secondary advantage is that IBDC is much more computationally efficient. This is because the time derivative approximation and the source are both isotropic and defined on the region mesh. This allows these terms to be pre-computed and combined into a transient source term, and the number of arithmetic operations performed per

\footnotetext{
${ }^{6}$ Prior to this work, the authors investigated other angular and spatial approximations to the angular flux time derivative (e.g. $P_{N}$ ) [22], which were intermediate in accuracy and computational expense to RBDC and IBDC.
} 
segment is reduced by about two thirds. Although the authors [22] and others [7],[8] have empirically confirmed that the accuracy of the isotropic approximation is acceptable for certain small problems, other researchers have encountered situations where the approximation is insufficiently accurate [3],[23], and the limits of its applicability are not adequately understood. IBDC1 is equivalent to Talamo's Method I [7].

\subsection{Source Derivative Propagation}

In light of the prohibitive memory expense of RBDC and the questionable limits of accuracy for IBDC, we developed a new approach to solving the time-dependent neutron transport equations. This method circumvents the necessity of storing the angular flux along the characteristics by defining an equation for the angular flux time derivative along the characteristic and solving that equation alongside the equation for the angular flux. This approach allows the angular flux time derivative to be recast principally in terms of the neutron source time derivative, and thus the method is called Source Derivative Propagation (SDP) when applied to MOC.

To derive the equation for the angular flux time derivative along the characteristic, we begin by taking a time derivative of Eq. (8) under the assumption that the cross sections are changing slowly (i.e.

$$
\begin{aligned}
& \left.\frac{d}{d t} \Sigma_{t r_{n},}^{r, n} \approx 0\right)^{7}:
\end{aligned}
$$

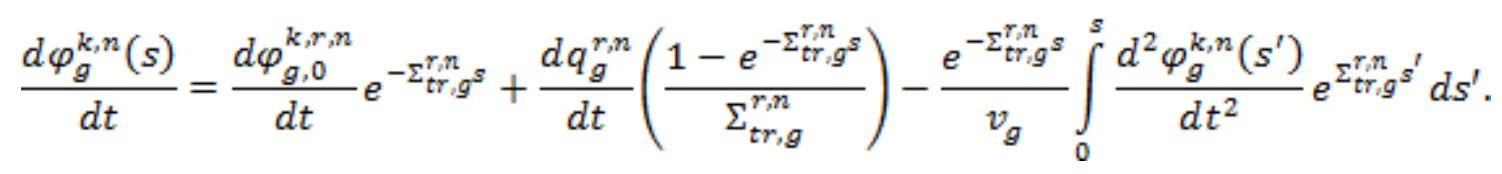

Equation (18) casts the angular flux time derivative along the characteristic in terms of the incoming angular flux time derivative, the source time derivative, and the second derivative of the

\footnotetext{
${ }^{7}$ While this approximation is valid throughout most of the space and time domain of the reactor transients we evaluated, we developed an alternate formulation that avoids this assumption by allowing the cross sections to vary linearly with time [13]. This alternate formulation was significantly more computationally expensive with a limited impact on accuracy.
} 
angular flux. Because we will propagate the angular flux time derivative along the characteristics, the incoming angular flux time derivative is known.

The source time derivative is approximated using BDF:

$$
\frac{d q_{g}^{r, n}}{d t}=\sum_{i=0}^{O_{\mathrm{BDF}}} A^{i} q_{g}^{r_{g} n-i} .
$$

While we investigated a range of approximations for the second time derivative of the angular flux [13], this paper will focus on two approximations. For the first approximation we assume that the second derivative of the scalar flux was very small and thus can be truncated:

$$
\frac{d^{2} \varphi_{g}^{k_{2} n}\left(s^{\prime}\right)}{d t^{2}} \approx 0 .
$$

This approximation (Truncated SDP or TSDP) was the focus of early efforts because the resulting method was derived through an alternate approach described in [15]. While this approximation was very accurate for the TWIGL transient described in section 3.1, it was insufficiently accurate for the C5G7 transient. In the second approximation, we assume that the second derivative was isotropic:

$$
\frac{d^{2} \varphi_{g}^{k_{2} n}\left(s^{\prime}\right)}{d t^{2}} \approx \frac{1}{4 \pi} \frac{d^{2} \phi_{g}^{r, n}}{d t^{2}} .
$$

This approximation (Isotropic SDP or ISDP) was more accurate for the C5G7 transient and produced nearly identical results to TSDP for the TWIGL transient. While we observed the ISDP method to be exclusively more accurate than TSDP, we have a more extensive data set of results for the TSDP method for the TWIGL transient, and thus we will use TSDP for the TWIGL results. In either case, as with IBDC, the SDP solution is not expected to converge to the transport solution as the time steps are refined.

To remain consistent with our use of BDF to treat the first time derivatives, we also approximated the second time derivative using backward differences [24]: 


$$
\frac{d^{2} \phi_{g}^{r, n}}{d t^{2}} \approx \sum_{i=0}^{o_{\mathrm{BDF}}+1} B^{i} \phi_{g}^{r, n-i}
$$

where the coefficients $B^{i}$ are a function of the previous time step sizes.

When these approximations are applied to Eq. (18), the remaining integral can be evaluated:

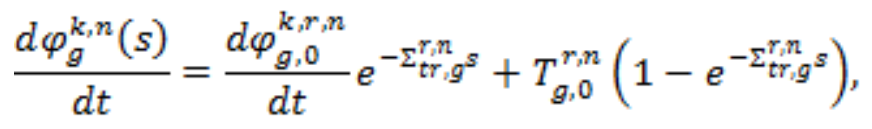

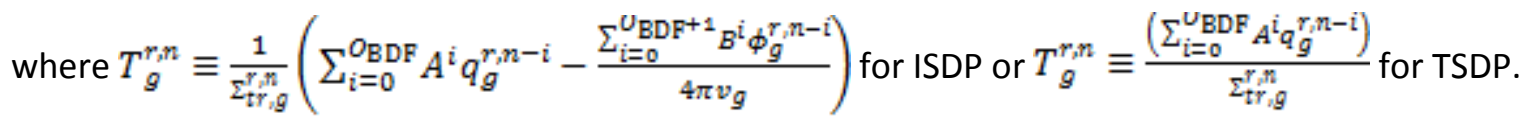

In both SDP methods, Eq. (23) is used to propagate the angular flux time derivative along the characteristics. To solve for the angular flux along the characteristic, we substitute Eq. (23) into Eq. (8) and evaluate the integral:

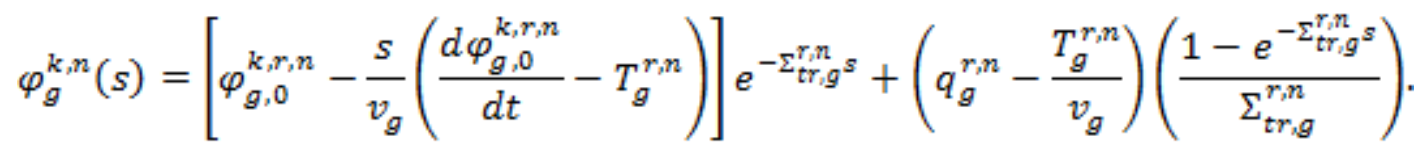

Equation (24) is used to propagate the angular flux along characteristics, serving a similar purpose to Eqs. (15) and (17) for RBDC and IBDC respectively. As for RBDC and IBDC, to calculate the region-wise scalar flux, Eq. (24) is integrated over space and angle within the region.

The algorithm for SDP is comparable to Tsujita's OTF method [8], but whereas SDP concurrently solves for the segment-wise angular flux and angular flux time derivative at the present time step, OTF concurrently solves for the segment-wise angular flux at the current and one or more previous time steps. A more detailed comparison of SDP and OTF is provided in [13].

\section{Test Problems and Numerical Results}

We empirically evaluated the accuracy and efficiency of SDP by comparison to the conventional MOC methods RBDC and IBDC. We implemented these methods in the computer code DeCART and used them to model two reactor transients. The first transient uses the comparatively simple two group 
TWIGL problem [17] while the second transient uses a modified C5G7 benchmark [13],[18]. Both transients are driven exclusively by changing cross sections without thermal feedback. The two transients were also modeled in two dimensions (2D).

For these transients, RBDC is employed in two different ways. First, we use the first-order RBDC (RBDC1) with a fine time step to generate a primary reference solution for each transient. This solution is used to verify that the SDP methods converge as the time step size decreases and the order of the time derivative approximation increases. Second, we use RBDC with various orders and time step sizes to determine whether the SDP methods replicate the RBDC solution for the same order and time step size. In other words, we will compare the solution of RBDCN with time step $\Delta t$ to SDPN with the same time step.

While the RBDC methods provide a reference solution for assessing accuracy, the IBDC method provides a benchmark for computational efficiency. IBDC is memory-efficient because it does not require storing angularly-dependent data, and it is computationally efficient because it does not require any additional arithmetic operations at the segment-level relative to steady-state MOC. This is in contrast to RBDC which requires the formation of a unique angular flux time derivative for every segment. We assess the efficiency of SDP by comparing the run-time and memory requirements of the methods.

\subsection{TWIGL Transient}

\subsubsection{TWIGL Benchmark Specification}

The TWIGL reactor is a 2D seed-and-blanket geometry $1.6 \mathrm{~m}$ along each side. It is surrounded by a vacuum and typically modeled with one-quarter or one-eighth core symmetry. The problem geometry is displayed in Figure 2. The problem specifies one delayed neutron group and cross sections for two energy groups within three different material regions: Regions 1 and 2 are seed regions while Region 3 is 
the blanket region. The transient was driven by varying the thermal absorption cross section in Region $1^{8}$.

Table 1 provides both the TWIGL reactor macroscopic cross sections by region and the kinetics parameters. The arrows in the table indicate the cross sections that are linearly-ramped over $0.2 \mathrm{~s}$.

Table 1. TWIGL cross sections and kinetics parameters

\begin{tabular}{c|c|c|c|c|c|c|}
\hline Region & Group, $g$ & $\begin{array}{c}\Sigma_{a} \\
\left(\mathrm{~cm}^{-1}\right)\end{array}$ & $\begin{array}{c}v \Sigma_{f} \\
\left(\mathrm{~cm}^{-1}\right)\end{array}$ & $\begin{array}{c}\Sigma_{t} \\
\left(\mathrm{~cm}^{-1}\right)\end{array}$ & $\begin{array}{c}\Sigma_{s, g \rightarrow g} \\
\left(\mathrm{~cm}^{-1}\right)\end{array}$ & $\begin{array}{c}\Sigma_{s, g \rightarrow g^{\prime}} \\
\left(\mathrm{cm}^{-1}\right)\end{array}$ \\
\hline \multirow{2}{*}{1} & 1 & 0.01 & 0.007 & 0.238095 & 0.218095 & 0.01 \\
& 2 & $0.15 \rightarrow 0.1465$ & 0.2 & $0.83333 \rightarrow 0.82983$ & 0.68333 & 0.0 \\
\hline \multirow{2}{*}{2} & 1 & 0.01 & 0.007 & 0.238095 & 0.218095 & 0.01 \\
& 2 & 0.15 & 0.2 & 0.83333 & 0.68333 & 0.0 \\
\hline \multirow{2}{*}{3} & 1 & 0.008 & 0.003 & 0.25641 & 0.23841 & 0.01 \\
& 2 & 0.05 & 0.06 & 0.666667 & 0.616667 & 0.0 \\
\hline
\end{tabular}

We modeled the TWIGL reactor in DeCART using quarter-core symmetry with 100 square "assemblies" that were $32 \mathrm{~cm}$ on each side. These assemblies contained $4 \times 4$ cells that were $2 \mathrm{~cm}$ long and contained 25 square flat-source regions each. The MOC ray spacing was $0.02 \mathrm{~cm}$, and the number of azimuthal and polar angles in $90^{\circ}$ were 8 and 4 respectively. The initial power was normalized to one.

\subsubsection{Steady-State Eigenvalue Solution}

The TWIGL transient begins from an assumed steady-state critical condition with the state initialized using an eigenvalue problem. We calculated the eigenvalue to be $k_{\text {eff }}=0.91605$ which was in good agreement with the results reported using VARIANT-K [29] (0.91609). The steady-state power distribution is presented in Figure 3.

\subsubsection{Linear Ramp Transient}

For the TWIGL ramp transient, the thermal absorption cross section in Region 1 is linearly decreased from $0.15 \mathrm{~cm}^{-1}$ to $0.1465 \mathrm{~cm}^{-1}$ over $0.2 \mathrm{~s}$. This results in a slow exponential power increase

\footnotetext{
${ }^{8}$ A TWIGL step-change transient was also modeled in [13]. The performance of the SDP methods for the stepchange transient were similar and intermediate to the results for the TWIGL ramp and the C5G7 transient.
} 
which can be accurately modeled with a very large range of time steps, which is useful for error scaling. Note that the original specification of the TWIGL ramp transient involved modeling the transient out to $0.5 \mathrm{~s}$ even though the linear ramp ends at $0.2 \mathrm{~s}$. We stopped the transient to $0.2 \mathrm{~s}$ to limit the computational requirements of the model.

The reference solution used RBDC1 with 0.01 ms time steps. RBDC1 requires approximately 4 GB of RAM to store the angular fluxes for the current and previous point in time. A power trace for the TWIGL core is presented in Figure 4, with the final relative power of 1.980412. The reference solution required approximately 20 hours using eight $2.53 \mathrm{GHz}$ Intel Xeon processors.

We assessed whether SDP1 converges to the reference solution as the time step size decreases. To limit the sheer amount of data in each of the solutions while still being able to compare the results for many different time step sizes, we used the final power for the core at the end of the transient for comparison. Specifically, we compared the absolute magnitude of the relative difference in the final power:

$$
\epsilon_{\mathrm{X}}(\Delta t)=\left|\frac{P_{\mathrm{X}}(\Delta t)-P_{\mathrm{RBDC} 1}(0.01 \mathrm{~ms})}{P_{\mathrm{RBDC} 1}(0.01 \mathrm{~ms})}\right|,
$$

where $P_{\mathrm{X}}(\Delta t)$ is the final power for method $\mathrm{X}$ with time step $\Delta t$.

In Figure 5 we plotted the error for SDP1 on a log-log plot as a function of the time step size. The time steps varied over three orders of magnitude. We see that the error in the SDP1 solution declines as the time step size decreases, indicating that the SDP1 method is converging to the reference solution. The slope of the convergence is 0.951 .

Next we evaluated whether the high-order SDP methods converge to the reference solution as the order increases, and as the time step size decreases. Figure 6 shows the final power for SDP1-4 as a function of time step as well as the reference solution; SDP5-6 is suppressed to limit clutter. Each method converges downward as the time step is refined, with SDP2-6 converging to 1.980396, which is 
slightly below the RBDC1 reference solution of 1.980412. The corresponding RBDC methods also converged to the same values.

In Figure 7 we plotted the error for SDP1-6 on log-log plot as a function of time step size. For large time steps we observe relatively linear convergence to the reference solution with slope that increases with the order of the method. However, we also observe a "dip" in error for each of the high order methods. Based on further investigation, we understand this crossover to correspond to the point which the high-order methods become more accurate than the reference solution. This can be confirmed by generating a reference solution with a yet smaller time step, but this required prohibitive run-times. However, we note that when the time step for the reference solution is adjusted upward (e.g. to $0.025 \mathrm{~ms}$ or $0.05 \mathrm{~ms}$ ) the transition point shifts upward as well, which is consistent with this explanation. Further, the slow, exponential power increase exhibited by the TWIGL transient is a phenomenon we would expect BDF to model accurately. Finally, we note that we observed this transition for the high-order RBDC and IBDC methods as well (e.g. in Figure 8, discussed below), indicating that it is not specific to the SDP methods.

Above the transition point, we see the expected convergence behavior. If we discard the data below the transition, we can calculate the order of temporal convergence for the high-order methods:

$$
S_{\mathrm{X}}\left(\Delta t_{i}\right)=\frac{\ln \epsilon_{\mathrm{X}}\left(\Delta t_{i}\right)-\ln \epsilon_{\mathrm{X}}\left(\Delta t_{i+1}\right)}{\ln \Delta t_{i}-\ln \Delta t_{i+1}}
$$

where $\Delta t_{i}$ is one time step size and $\Delta t_{i+1}$ is the next smaller time step. Table 2 shows that, above the transition point, the slopes of convergence for the SDP methods are close to their theoretical order. There was insufficient data to estimate a slope for SDP6.

Table 2. Logarithmic error slopes for SDP methods discarding data below transition

\begin{tabular}{|c|c|c|c|c|c|c|}
\hline Method, $\mathrm{X}$ & SDP1 & SDP2 & SDP3 & SDP4 & SDP5 & SDP6 \\
\hline Slope, $S_{\mathrm{X}}$ & 0.95 & 1.91 & 2.92 & 4.09 & 5.30 & - \\
\hline
\end{tabular}


Next we compared the high-order SDP methods with corresponding RBDC methods of the same order and time step size to determine whether the SDP methods were reproducing the RBDC methods. Figure 8 shows the relative error for the high-order RBDC methods plotted as dashed lines with the error for the high-order SDP methods plotted as symbols in the same color as Figure 7. The error is nearly identical for each order and time step size because the RBDC and SDP methods are in excellent agreement. Thus, the propagated angular flux time derivative based on neutron source derivatives is a good approximation for the finite-differenced angular flux time derivative in RBDC, even when high order BDF approximations are used. This also confirms that the "error transition" in Figure 7 is not unique to the SDP methods, but is also observed for the high-order RBDC methods.

To further illustrate the agreement between SDP and RBDC, we compare the absolute difference in the final power:

$$
\epsilon_{\mathrm{abs}, \mathrm{XN}}(\Delta t)=\left|P_{\mathrm{RBDCN}}(\Delta t)-P_{\mathrm{XN}}(\Delta t)\right|
$$

where $\mathrm{X}$ is the method in question (e.g. SDP) and $N$ is the order of the method.

Figure 9 and Figure 10 present the absolute difference in final power as a function of time step size for the odd- and even-ordered methods respectively. In addition, the difference for IBDC1 is also provided for comparison. The SDP methods more accurately replicated the RBDC solutions with the same time step size than IBDC, although the error for IBDC is very small nonetheless.

We also assessed the spatial accuracy of the methods by comparing the relative pin power at the end of the transient when the same time step is used:

$$
\epsilon_{\mathrm{rel}, x y, \mathrm{X} 1}=\frac{\left|P_{x y_{2} \mathrm{RBDC} 1}-P_{x y, \mathrm{X} 1}\right|}{P_{x y_{2} \mathrm{RBDC} 1}}
$$

where $P_{x y \mathrm{x} 1}$ is the relative pin power at the end of the transient for pin location $x y$ using method X1 with a time step size of $1 \mathrm{~ms}$. 
The relative error in the pin power in the southeast quadrant for SDP1 is presented in Figure 11. For most pins the error is "zero" because the SDP results were identical to the RBDC results to the last decimal edited by DeCART $\left(1 \times 10^{-7}\right)$. The largest relative error is scattered in the outer blanket because these cells have a small absolute power. The largest absolute error occurred in the ramped seed region, where the power was slightly over-predicted, indicating that the propagated angular flux time derivative was slightly low. The error was small in the highest power cells in the seed near the inner blanket. We observed similar results for the higher order SDP methods as compared to the corresponding high order RBDC method, as well as for other time step sizes.

For comparison, Figure 12 provides the relative error in the pin power for IBDC1. Note that the scale is an order of magnitude larger than Figure 11. The relative error for IBDC1 is greatest at the core periphery where the power is low. However, there is also substantial error at the interfaces between the seed and blanket regions-including the peak power cells-which is typical of diffusion approximations. The error is nonetheless small throughout the geometry.

There are substantial differences in the computational and memory requirements of the SDP, RBDC, and IBDC methods. Table 3 provides the relative run-time and memory requirements for selected methods with a $0.5 \mathrm{~ms}$ time step using eight Intel Xeon processors. The ratio of these values closely correspond to the ratio of arithmetic operations required per segment for each method in MOC. A significant advantage of SDP (and IBDC) is that the run-time does not substantially increase when highorder BDF is used to approximate the time derivatives. By contrast, the run-time for high-order RBDC methods increases substantially over RBDC1.

The RBDC methods also require substantially more memory than the SDP and IBDC methods because of the extremely large number of angular fluxes that are stored to represent the time derivative. When high-order BDF is used to approximate the time derivative, the memory requirement for RBDC increases proportionally. By contrast, the memory requirements for high-order SDP (and IBDC) 
only increase modestly over their first-order versions. Similar trends were observed for the C5G7 transient.

Table 3. Run-time and memory requirements for TWIGL transient

\begin{tabular}{|l|c|c|}
\hline Methods & $\begin{array}{c}\text { Run-Time } \\
\text { (hr) }\end{array}$ & $\begin{array}{c}\text { Memory Required } \\
\text { (GB RAM) }\end{array}$ \\
\hline RBDC1 & 4.25 & 4 \\
\hline SDP1 & $\mathbf{2 . 4 6}$ & $\mathbf{0 . 0 9 2}$ \\
\hline IBDC1 & 1.31 & 0.080 \\
\hline RBDC6 & 6.78 & 14 \\
\hline SDP6 & $\mathbf{2 . 4 5}$ & $\mathbf{0 . 1 0 6}$ \\
\hline
\end{tabular}

\subsection{C5G7 Transient}

\subsubsection{C5G7 Benchmark Specification}

The C5G7 benchmark [18] depicts a small reactor with both $\mathrm{UO}_{2}$ and mixed oxide $\left(\mathrm{MOX}^{9}\right)$ fuel assemblies. Compared to TWIGL, the C5G7 geometry is more complex because it explicitly represents that cylindrical fuel rods rather than homogenizing assemblies. In addition, the C5G7 transient is more challenging because it is initiated with a step change rather than a linear ramp. While C5G7 was originally specified as a steady-state benchmark, we used a modified C5G7 control rod ejection transient [16], adapted from the transient specified by Tsujita [8].

C5G7 consists of eight $\mathrm{UO}_{2}$ assemblies and eight MOX assemblies. These assemblies are 21.42 $\mathrm{cm}$ square, and the reactor is surrounded by a water reflector $21.42 \mathrm{~cm}$ thick. Figure 13 shows the geometry used for the transient. The guide tubes of the four innermost assemblies include control rod drives (CRD), one of which is ejected (CRE) to drive the transient.

Each assembly consists of a $17 \times 17$ array of circular fuel pins and guide tubes which are surrounded by moderator. Figure 14 provides the pin cell layout for the southeast quadrant, excluding the CRD in the inner assembly. Each pin cell is $1.26 \mathrm{~cm}$ square, and the fuel pins have a radius of $0.54 \mathrm{~cm}$ centered on the pin cell.

\footnotetext{
${ }^{9} \mathrm{MOX}$ is nuclear fuel that contains plutonium oxide in addition to uranium oxide.
} 
C5G7 uses a seven-group cross section library which the designers selected because of its challenging structure. The cross sections and kinetics parameters are provided in [8]. Due to the limitations of the 2D transport method, the ejected control rod drive is removed instantaneously as a step change.

We modeled the C5G7 reactor using DeCART as a $12 \times 12$ grid of assemblies each consisting of an array of $17 \times 17$ pin cells. Each pin cell was decomposed into 32 regions as depicted in Figure 15 . For MOC we used a ray spacing of $0.04 \mathrm{~cm}$ with 8 azimuthal angles and 3 polar angles in $90^{\circ}$.

\subsubsection{C5G7 Steady-State Eigenvalue Solution}

The C5G7 transient begins from a normalized steady-state critical condition which is initialized using an eigenvalue problem. We calculated the eigenvalue to be $k_{\text {eff }}=1.148013$. Figure 16 shows the steady-state relative power distribution for the south-east quadrant of the core.

\subsubsection{C5G7 Transient}

The C5G7 transient is driven by replacing the control rod material in the guide tubes of the southeast, central $\mathrm{UO}_{2}$ assembly with the moderator-filled guide tube material. This causes a fast exponential increase in power. The transient was simulated for $0.05 \mathrm{~s}$, over which the power increases by two orders of magnitude. We generated the reference solution using RBDC1 with a 0.01 ms time step, which required about 3.5 days using eight $2.53 \mathrm{GHz}$ Intel Xeon processors and approximately $30 \mathrm{~GB}$ of RAM to store the angular flux. Figure 17 presents the reference solution power trace for the transient with a final power of 126.030 .

We assessed the convergence of the SDP methods by comparing their final power to that of the reference solution using Eq. (25). Figure 18 is a log-log plot of the relative error in the final power for the SDP methods as a function of time step size. Note that the C5G7 transient was amenable to a much narrower range of time step sizes than the TWIGL transient due to the rapid change in power. While the high order SDP methods are more accurate than the lower order methods, there are diminishing returns 
beyond second order. This is because the high-order BDF approximations require many previous data points to achieve high-order accuracy. Unlike the TWIGL ramp transient, this transient begins with a fast step change, and the error incurred in the early time steps can dominate the overall error, especially for longer time steps. This is why the error scaling for the high-order methods with large time steps is similar to the SDP2; similar trends were observed for a TWIGL step change transient [13].

We also modeled the transient using RBDC methods up to order three; higher order RBDC methods had excessive memory requirements (+15 GB RAM per additional BDF order). Figure 19 shows the relative error for SDP1-3 and RBDC1-3. As for the TWIGL transient, the SDP solutions for the C5G7 case closely track the RBDC solutions with the same order and time step, again confirming that the propagated angular flux time derivative is a good approximation for the finite-differenced angular flux derivative.

In Figure 20 we directly compare the solutions for SDP and RBDC with the same order and time step using Eq. (27). A comparison of IBDC1 and RBDC1 is also included. In contrast to the TWIGL transient, the improvement in accuracy of the SDP methods over IBDC is modest when comparing the total core power. This appears to be due to the fact that the C5G7 transient is faster and exhibits a larger second time derivative than the TWIGL transient. Nonetheless, the SDP method is uniformly more accurate than IBDC for this transient.

We also assessed the accuracy of the spatial variation of the power for each of the methods. Figure 21 shows the spatially-dependent relative error in the relative pin power for SDP1 as compared to RBDC1 with the same $1 \mathrm{~ms}$ time step at the end of the transient. The error is small throughout the spatial domain, including within the rodded and MOX assemblies, indicating that the SDP method accurately captured the spatial variation of the RBDC solution. Similar trends were observed when the high-order SDP methods were compared to similar order RBDC methods. 
The spatially-dependent error in the relative pin power for IBDC1 is presented in Figure 22. Note that the scale for this plot is an order of magnitude larger than that of Figure 21, and the error is large near the assembly with the ejected control rods. This indicates that the SDP methods more accurately captured the spatial variation in the power than IBDC did, although IBDC and SDP exhibited comparable error in the total power. In light of these results, we are investigating alternative treatments for the second derivative term in SDP.

\section{Summary and Conclusion}

In this work, we developed a new time-dependent method of characteristics (MOC) formulation that treats the angular flux time derivative by propagation along characteristics in terms of neutron source derivatives called source derivative propagation (SDP). SDP was developed in light of the challenges introduced by conventional time integration techniques for time-dependent neutron transport. Conventional techniques require storing the angular flux, which requires prohibitively large memory for large problems. The SDP method circumvents this issue by casting the angular flux time derivative in terms of the propagated effects of the neutron source derivatives along the characteristics. An additional benefit of SDP is that it inexpensively permits high order time integration using BDF.

We empirically evaluated the accuracy of the SDP methods using DeCART with two test transients: the comparatively easy TWIGL transient and the more complex C5G7 transient. We confirmed that the SDP method converged for each transient as the time step size was decreased and the order was increased. We also observed that the SDP methods accurately replicated the reference method solution when the same order and time step was used. However, while the SDP methods were substantially more accurate than the IBDC methods for the TWIGL transient, they were only modestly more accurate for the C5G7 transient. Nonetheless, both the SDP and IBDC methods accurately replicated the reference solution and were substantially more computationally and memory efficient than RBDC. 
The SDP methods in this work were investigated because they have a convenient mathematical form which allows time derivatives to be efficiently represented using an isotropic neutron source derivative that is spatially invariant within the flat source region. However, if we allow the neutron source to vary spatially within regions, the mathematical form of SDP will become substantially more complex. This is also true if we use high-order moments of the angular flux to model anisotropic scattering, although we note that high-order moments could also be used to improve the treatment of the second derivative of the angular flux. Nonetheless, the SDP methods show good promise generating high fidelity reactor kinetics solutions, and in light of this we are investigating further options for angular flux time derivative propagation.

\section{Acknowledgements}

The authors acknowledge Han Joo, Ben Collins, Mathieu Hursin, and Brendan Kochunas for their assistance understanding and modifying the DeCART source code. This research was supported by the Consortium for Advanced Simulation of Light Water Reactors (www.casl.gov), an Energy Innovation Hub (http://www.energy.gov/hubs) for Modeling and Simulation of Nuclear Reactors under U.S. Department of Energy Contract No. DE-AC05-00OR22725. 


\section{References}

[1] T. M. SUTTON and B. N. AVILES, "Diffusion Theory Methods for Spatial Kinetics Calculations," Prog. Nucl. Energy, 30, 119-182 (1996).

[2] S. GOLUOGLU and H. L. DODDS, "A Time-Dependent, Three-Dimensional Neutron Transport Methodology," Nucl. Sci. Eng., 139, 248-261 (2001).

[3] A. PAUTZ and A. BIRKHOFER, "DORT-TD: A Transient Neutron Transport Code with Fully Implicit Time Integration," Nucl. Sci. Eng., 145, 299-319 (2003).

[4] J. Y. CHO et al., "Transient Capability for a MOC-Based Whole Core Transport Code DeCART," Trans. Am. Nucl. Soc., 92, 721 (2005).

[5] J. B. TAYLOR and A. J. BARATTA, "A Time-Dependent Method of Characteristics for 3D Nuclear Reactor Kinetics Applications," Proc. Intl. Conf. on Math. and Comput. Methods Applied to Nucl. Sci. Eng. (M\&C2009), Saratoga Springs, USA, May 3-7 (2009).

[6] A. SEUBERT, "The Time-Dependent 3-D Transport Code TORT-TD: Recent Advances and Applications," Trans. Am. Nucl. Soc., 104, 879-882 (2011).

[7] A. TALAMO, "Numerical Solution of the Time Dependent Neutron Transport Equation by Method of Characteristics," J. Comput. Phys., 240, 248-267 (2013).

[8] K. TSUJITA, et al., "Higher Order Treatment on Temporal Derivative of Angular Flux for Time-Dependent MOC," Proc. Intl. Conf. on Math. and Comput. Methods Applied to Nucl. Sci. Eng. (M\&C2013), Sun Valley, USA, May 5-9 (2013).

[9] J. R. ASKEW, "A Characteristics Formulation of the Neutron Transport Equation in Complicated Geometries," AAEW-M 1108, United Kingdom Atomic Energy Authority (1972).

[10]M. J. HALSALL, "Cactus, A Characteristic Solution to the Neutron Transport Equations in Complicated Geometries," AEEW-R-1291, United Kingdom Atomic Energy Authority (1980).

[11]H. G. JOO et al., "Methods and Performance of a Three-dimensional Whole-core Transport Code DeCART," Proc. PHYSOR2004, Chicago, USA (2004).

[12]M. HURSIN et al., "DeCART v2.05 Theory Manual," (2008).

[13]T. M. PANDYA and M.L. ADAMS, "Method of Long Characteristics Applied in Space and Time," Proc. Intl. Conf. on Math. and Comput. Methods Applied to Nucl. Sci. Eng. (M\&C2009), Saratoga Springs, USA, May 3-7 (2009).

[14]K. TSUJITA, T. ENDO, and A. YAMAMOTO, "Kinetic Calculation Method in Space-Time Frame using Characteristic Line," Trans. Am. Nucl. Soc., 106, 743-746 (2012).

[15]A. J. HOFFMAN and J. C. LEE, "A Variable-Order Time-Dependent Neutron Transport Method for Nuclear Reactor Kinetics using Analytically-Integrated Space-Time Characteristics," Proc. Intl. Conf. on Math. and Comput. Methods Applied to Nucl. Sci. Eng. (M\&C2013), Sun Valley, USA, May 5-9 (2013).

[16]A. J. HOFFMAN, "A Time-Dependent Method of Characteristics Formulation with Time Derivative Propagation," Dissertation, University of Michigan Department of Nuclear Engineering and Radiological Sciences, (2013). 
[17]J. B. YASINSKY and A. F. HENRY, "Some Numerical Experiments Concerning Space-Time Reactor Kinetics Behavior," Nucl. Sci. Eng., 22, 171 (1965).

[18]E. LEWIS et al., "Benchmark Specification for Deterministic 2D/3D MOX Fuel Assembly Transport Calculations without Spatial Homogenization," Nuclear Energy Agency (2001).

[19]D. GINESTAR et al., "High Order Backward Discretization of the Neutron Diffusion Equation," Ann. Nucl. Energy, 25, 47-64 (1998).

[20]V. M. GARCIA et al., "Parallel Resolution of the Two-Group Time Dependent Neutron Diffusion Equation with Public Domain ODE Codes," Lecture Note in Computer Science, 3402, 457-497 (2005).

[21]C. B. SHIM, et al., "Application of Backward Differentiation Formula to Spatial Reactor Kinetics Calculation with Adaptive Time Step Control," Nucl. Eng. Tech., 43, 531-546 (2011).

[22]A. J. HOFFMAN and J. C. LEE, "Low-Order Approximations to the Angular Flux Time Derivative for Transport-based Reactor Kinetics," Trans. Am. Nucl. Soc., 108, 777-780 (2013).

[23]S. HAMILTON, C. DE OLIVEIRA, and K. T. CLARNO, "Error Control in a Time-Dependent Slice Balance Method," Trans. Am. Nucl. Soc., 97, pp. 533-535 (2007).

[24]B. FORNBERG, "Generation of Finite Difference Formulas on Arbitrarily Spaced Grids," Mathematics of Computation, 51, no. 184, 699-706, (1988).

[25]A. RINIEISKI et al., "Time-Dependent Neutron Transport with Variational Nodal Methods," Proc. Joint. Intl. Conf. Math. Methods and Supercomput. for Nucl. Appl., Saratoga Springs, USA (1997).

\section{Figures}

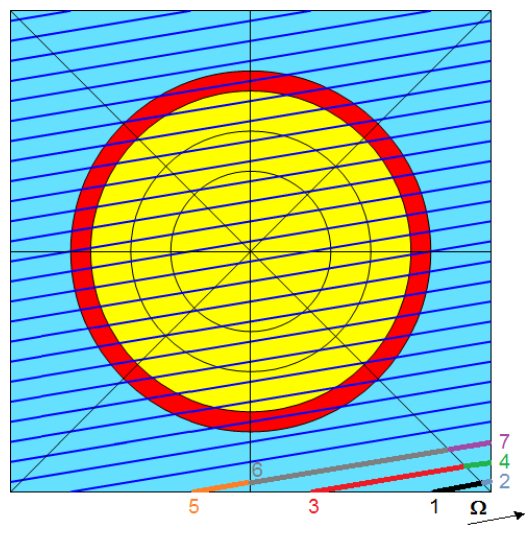

Figure 1. Example MOC meshing for a single fuel pin with characteristics at angle with seven segments labeled. 


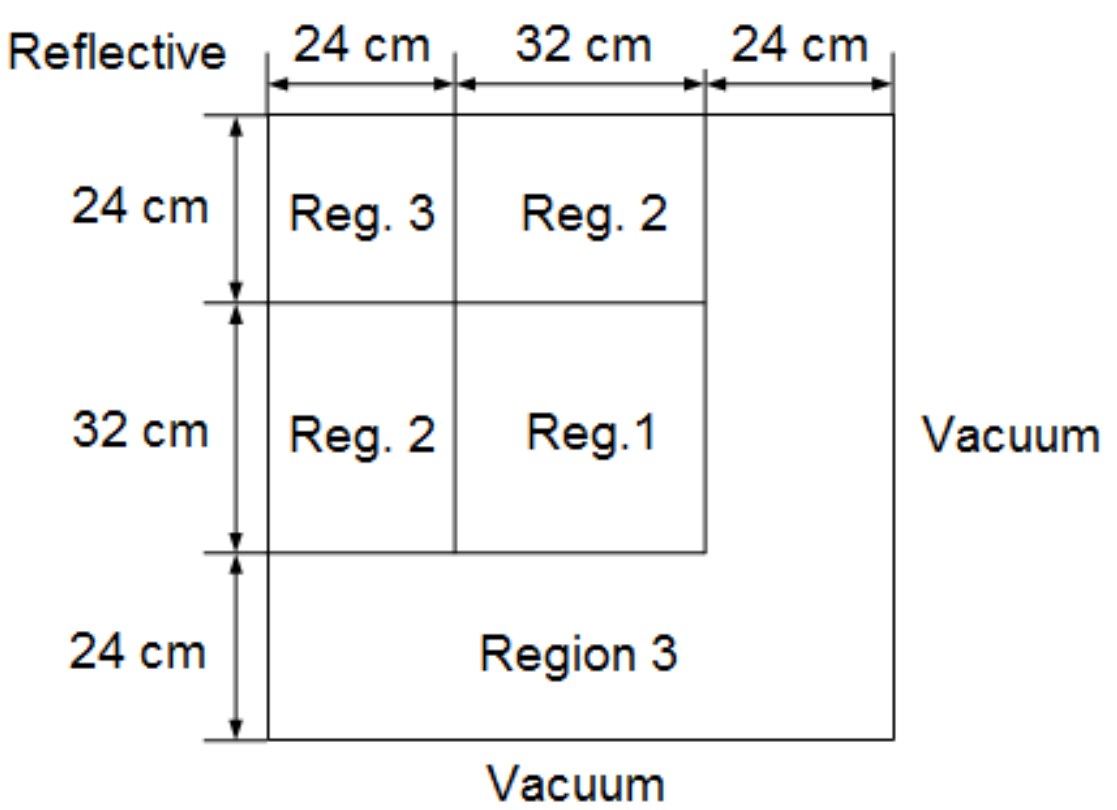

Figure 2. Southeast quadrant of TWIGL reactor
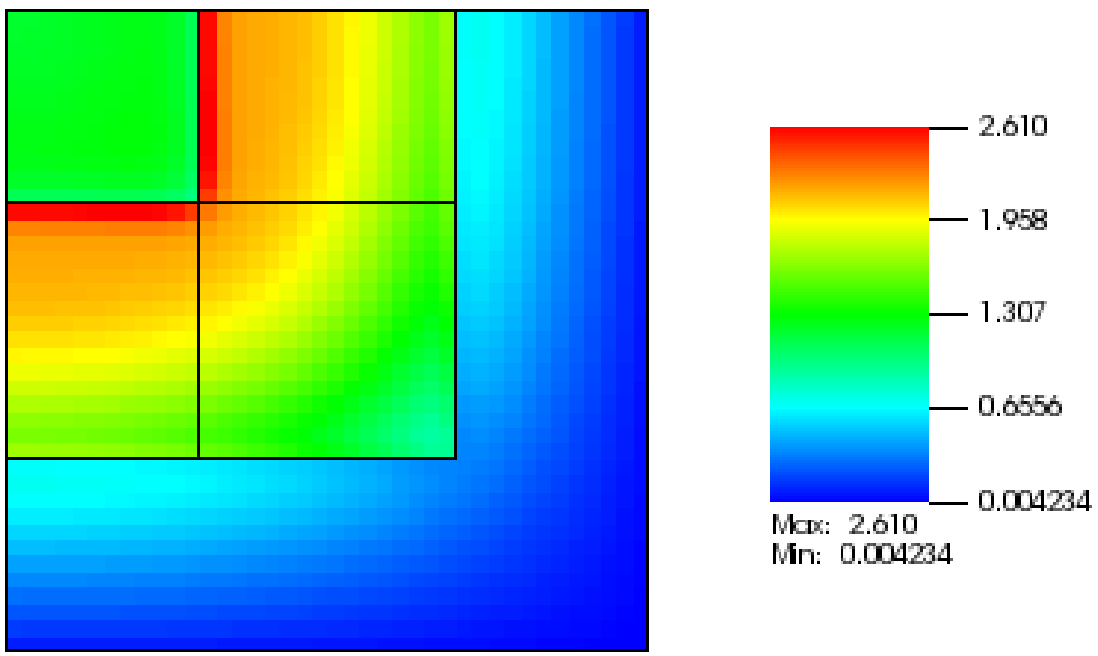

Figure 3. Steady-state relative power distribution for TWIGL 


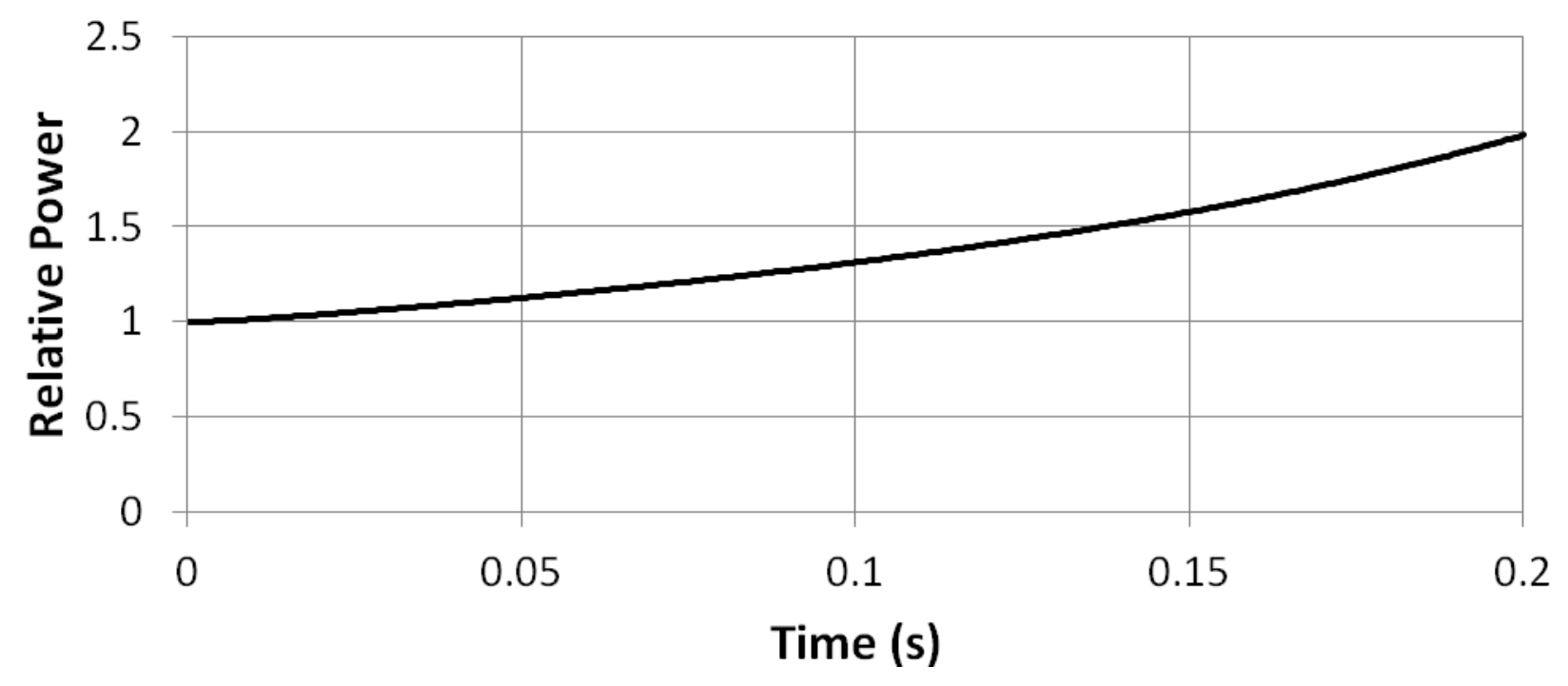

Figure 4. Relative core power for reference solution for TWIGL ramp transient

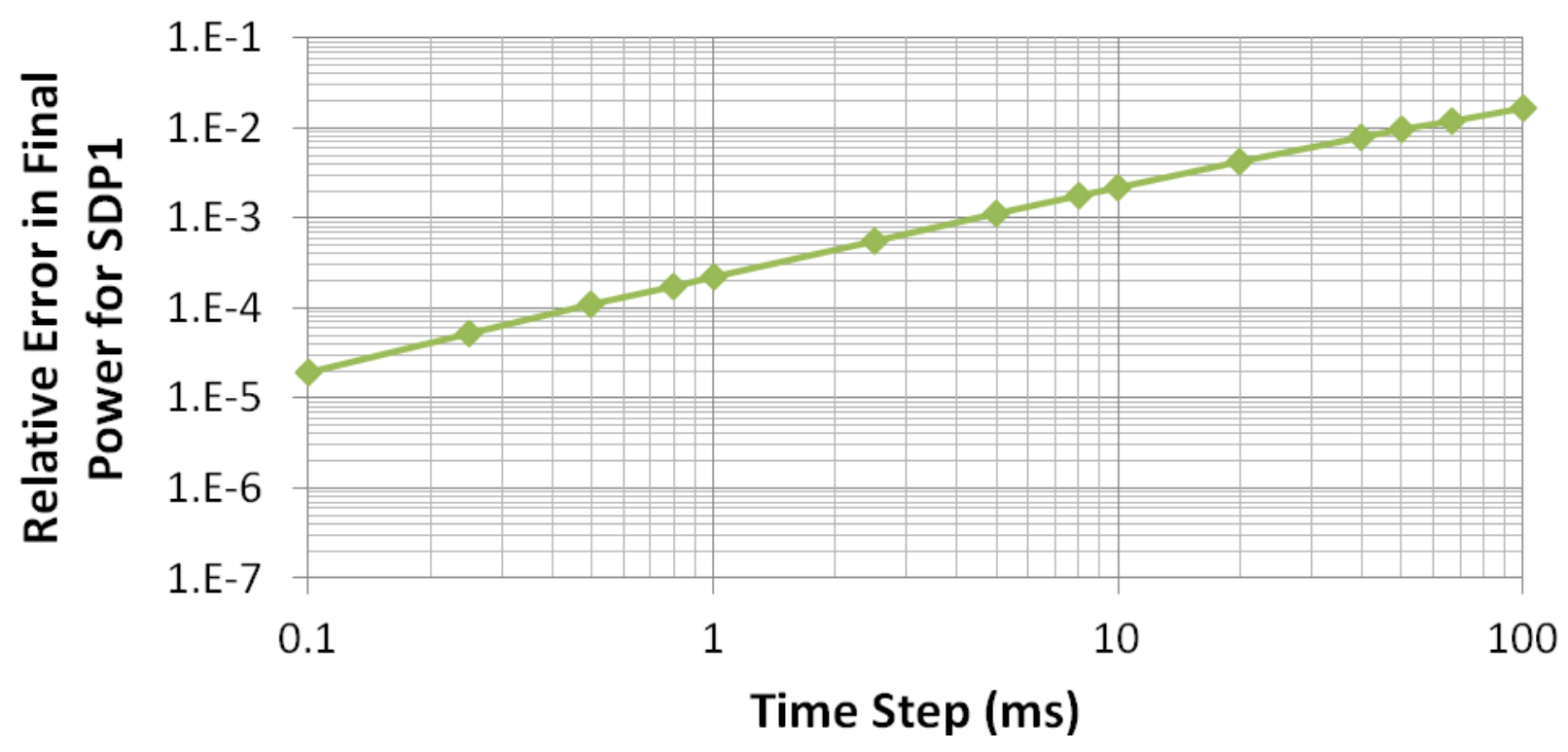

Figure 5. Relative error in the final power for SDP1 as a function of time step 


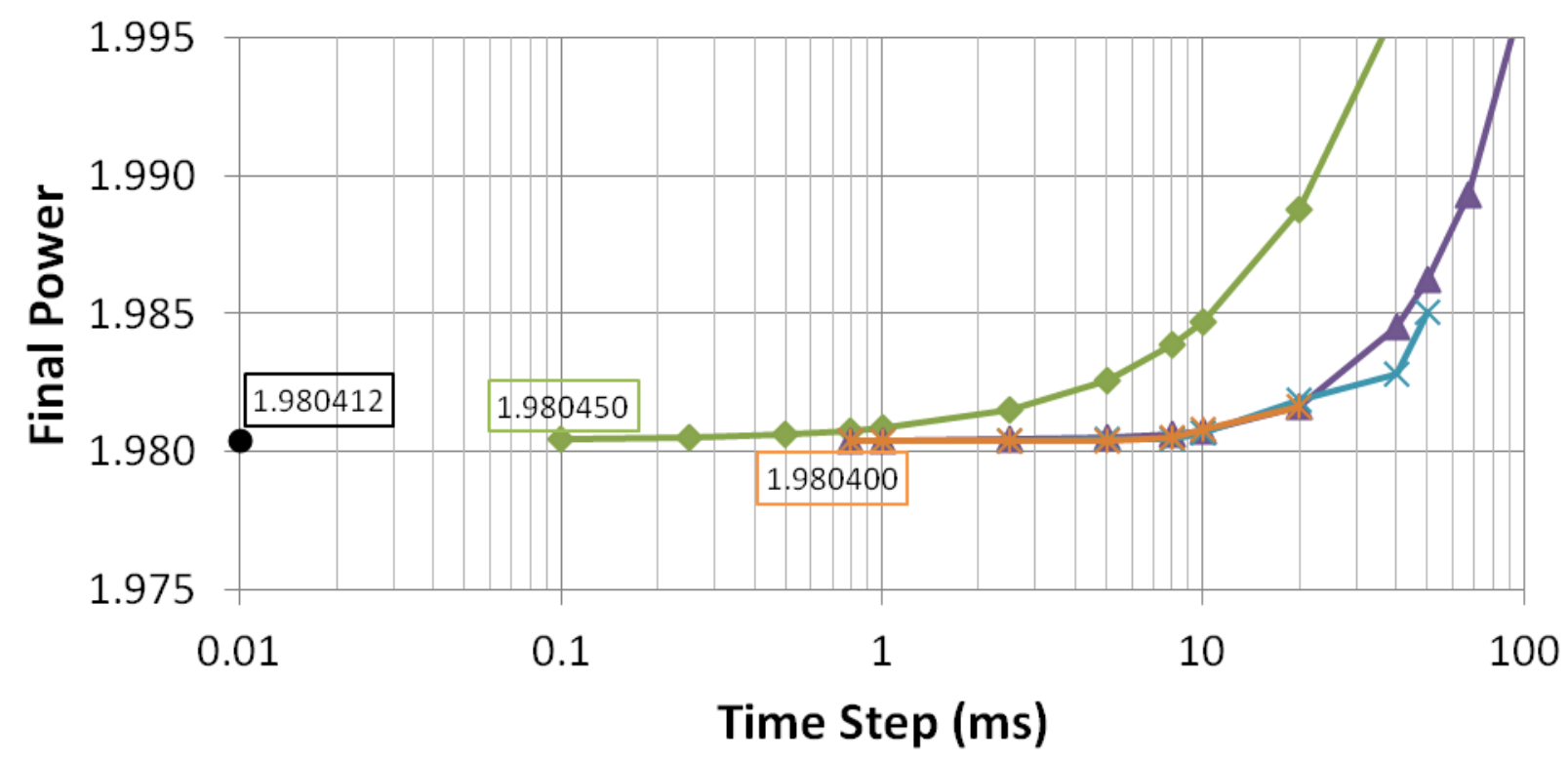

- Reference Solution $\multimap$ SDP1 $\leftarrow$ SDP2 $\leftarrow$ SDP3 $\leftarrow$ SDP4

Figure 6. Final power for SDP1-4 as a function of time step with labels for the reference solution, SDP1 with a $0.1 \mathrm{~ms}$ time step, and SDP with a $0.8 \mathrm{~ms}$ time step

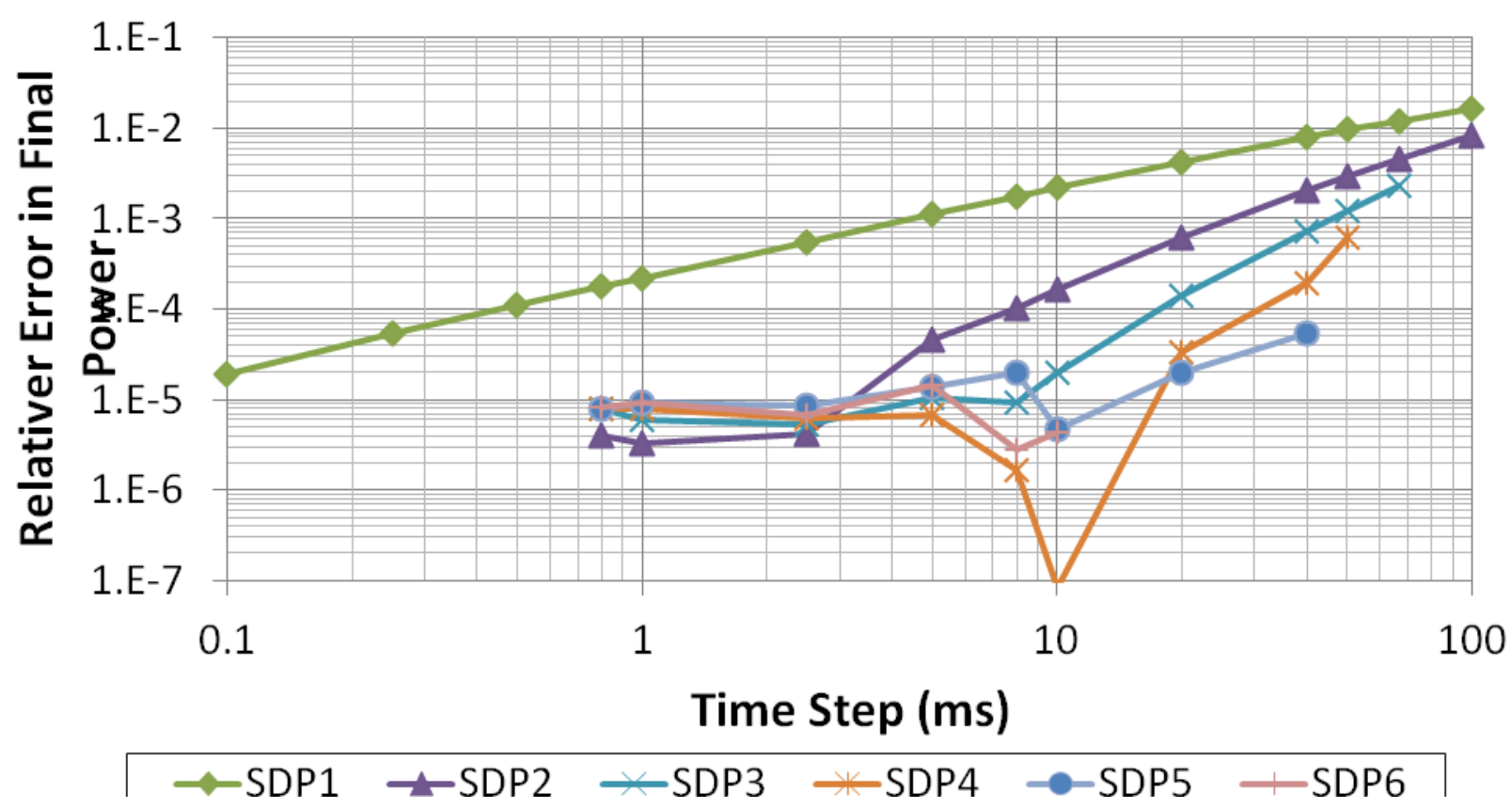

Figure 7. Relative error in final power for SDP1-6 as a function of time step. The apparent dip in error for the high-order methods corresponds to the crossover as the high-order methods converge to a lower solution than the reference solution, which is illustrated in Figure 6. 


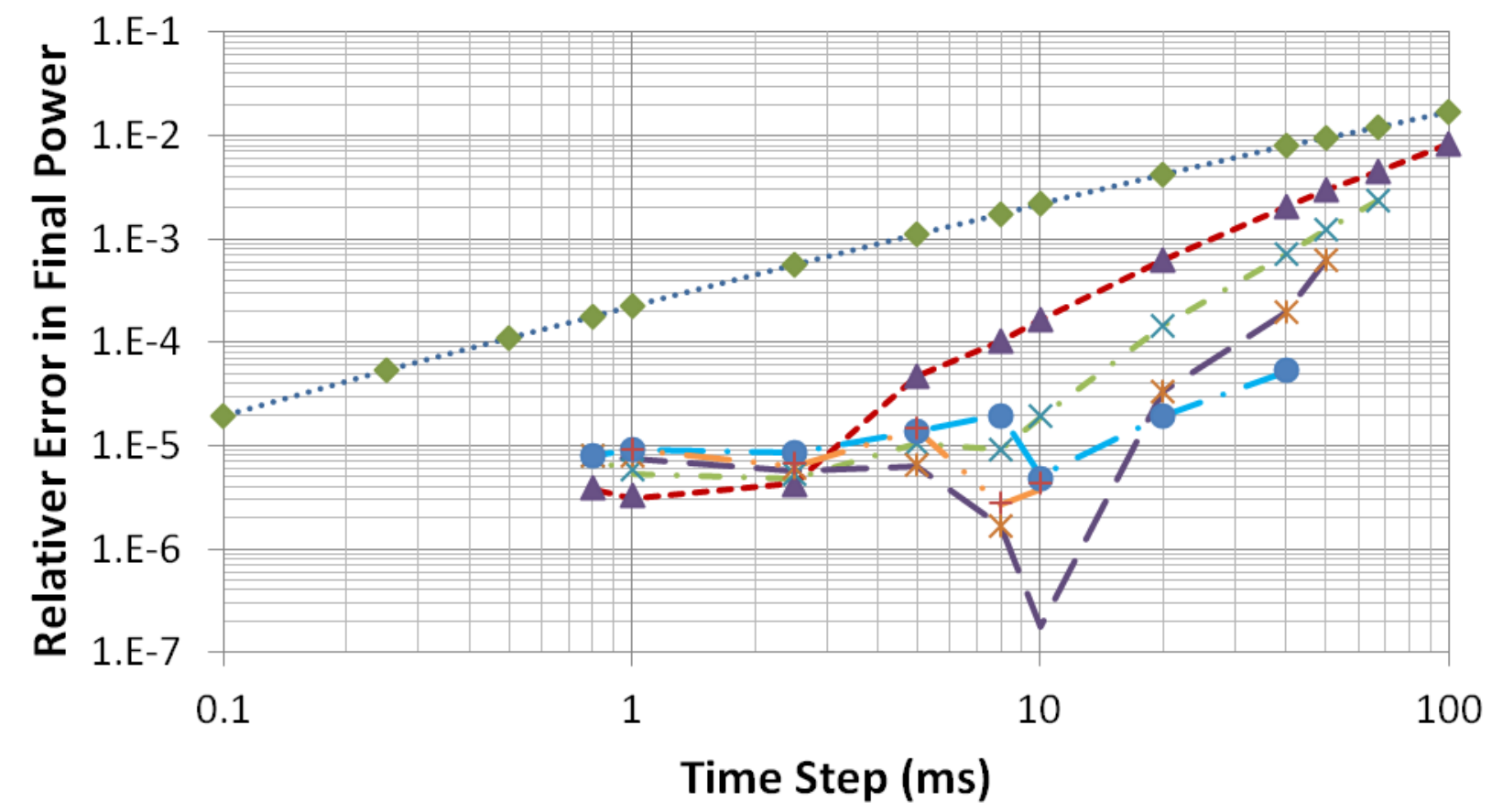

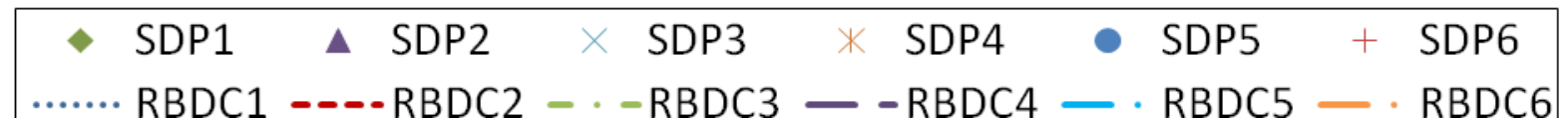

Figure 8. Relative error in final power for SDP1-6 and RBDC1-6 as a function of time step, illustrating that the SDP solutions closely track the corresponding RBDC solutions. 


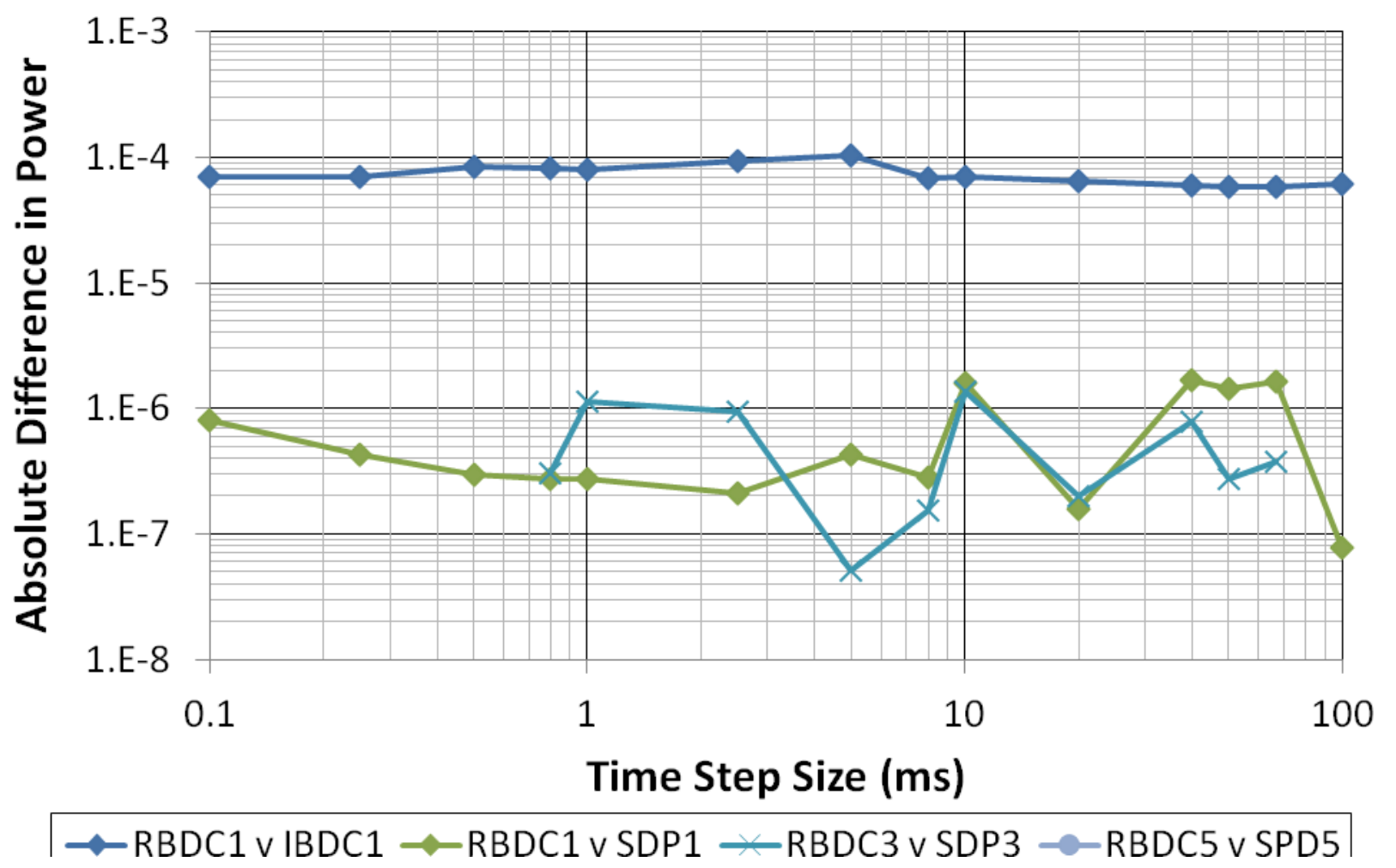

Figure 9. Absolute difference in relative final power: odd-ordered methods, illustrating the accuracy improvement of the SDP methods over IBDC.

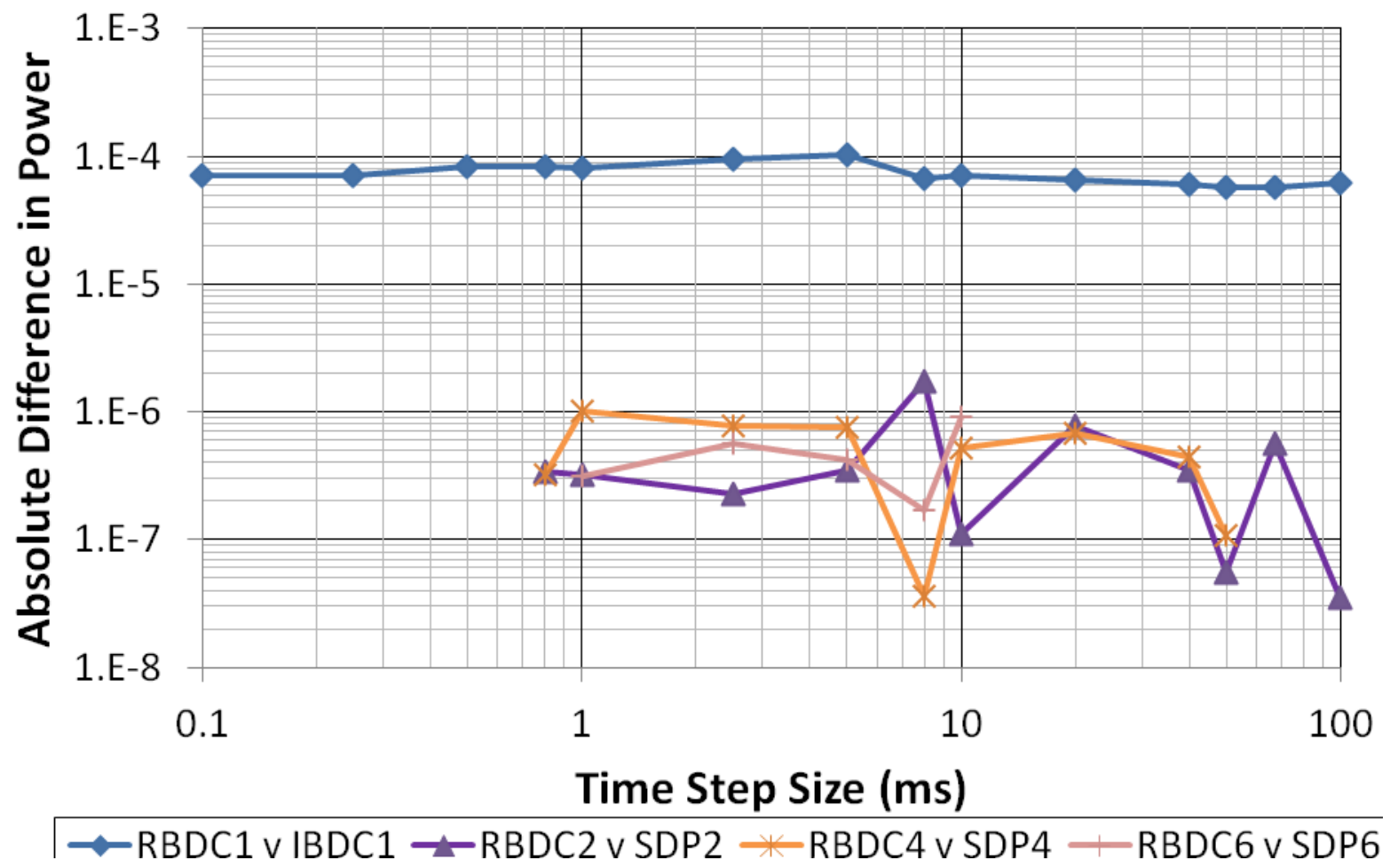

Figure 10. Absolute difference in relative final power: even-ordered methods, illustrating the accuracy improvement of the SDP methods over IBDC. 

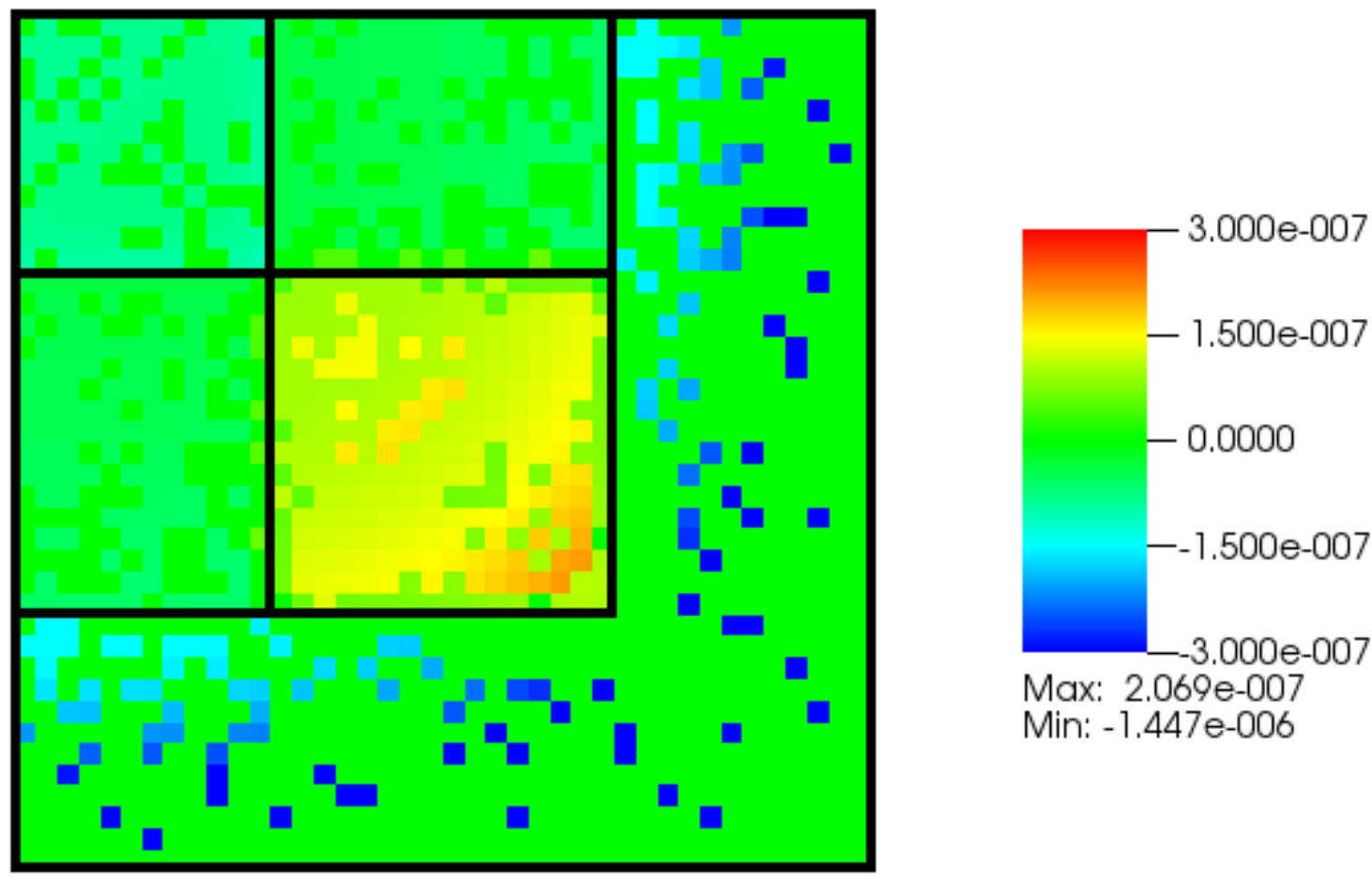

Max: $2.069 \mathrm{e}-007$

Min: - 1.447e-006

Figure 11. Relative error in the pin cell power for SDP1
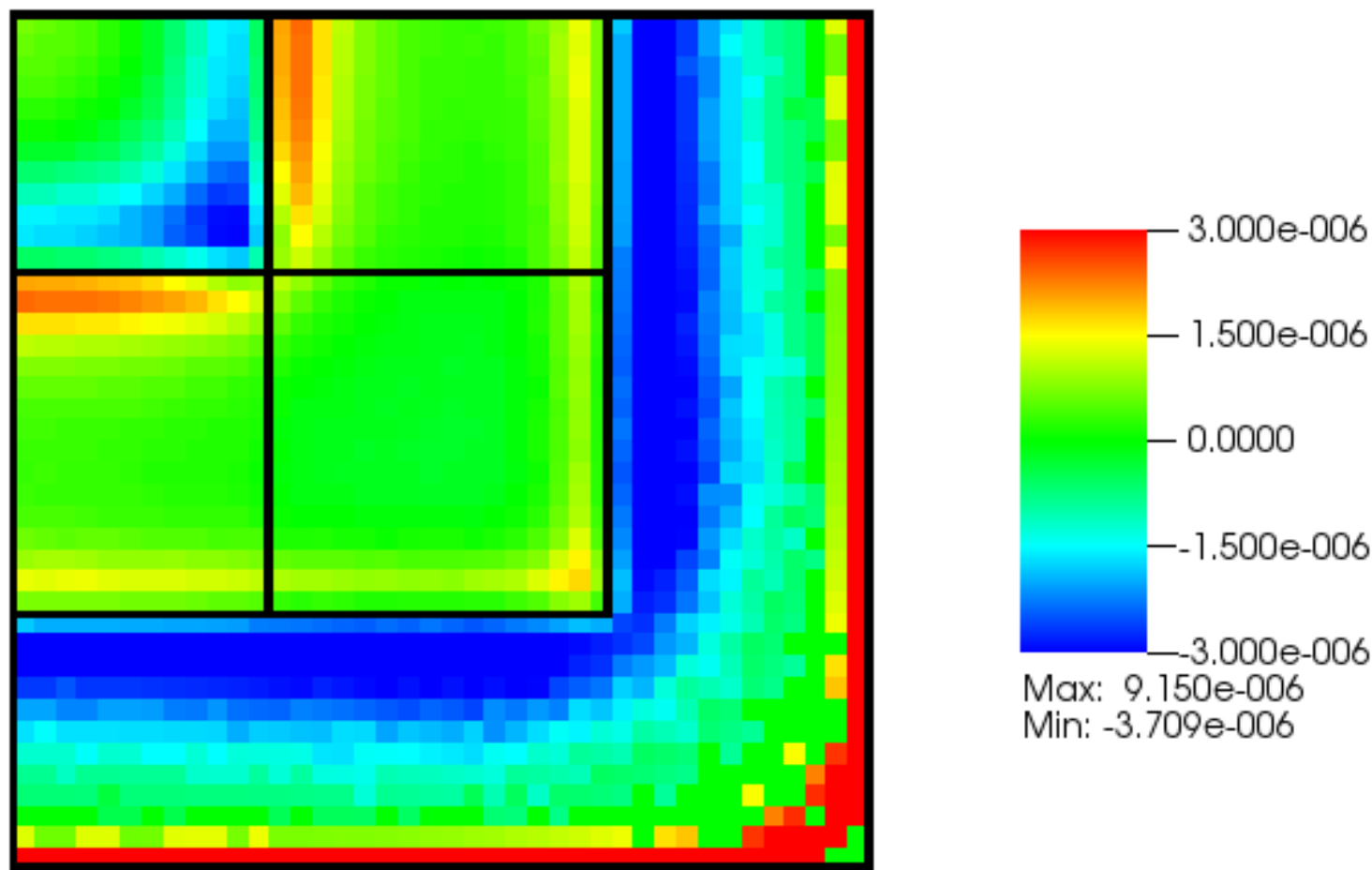

Max: $9.150 \mathrm{e}-006$ Min: -3.709e-006

Figure 12. Relative error in the pin cell power for IBDC1 


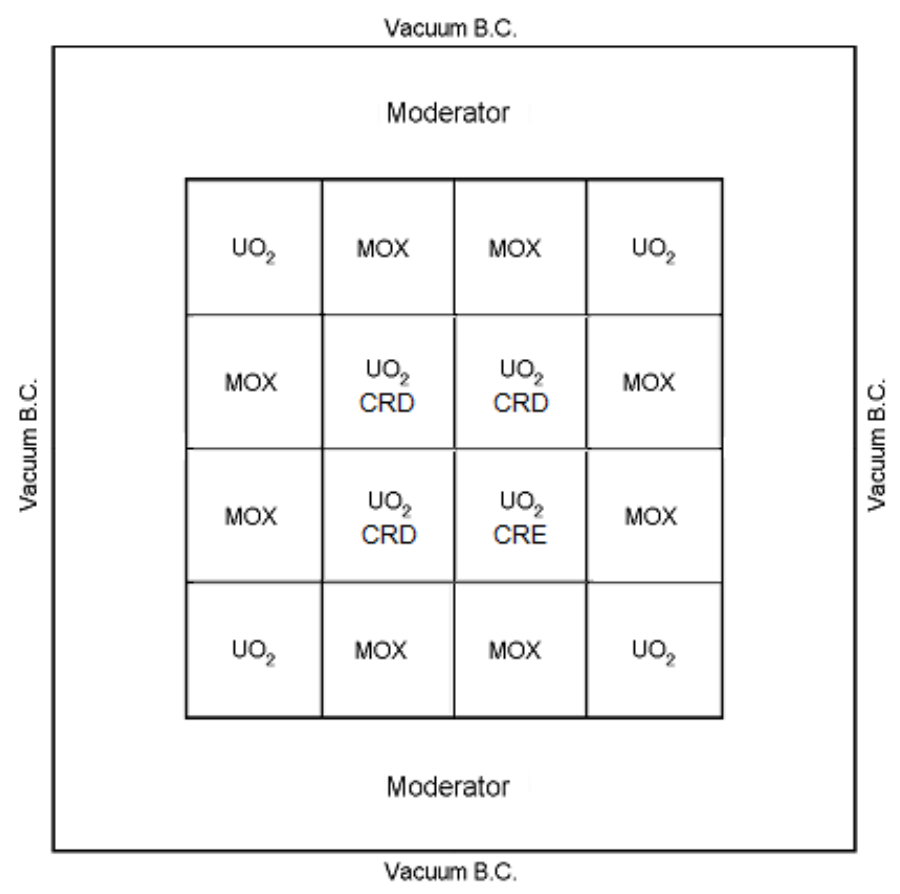

Figure 13. C5G7 reactor transient geometry by fuel assembly: $\mathrm{CRD}=$ Control Rod Drive, $\mathrm{CRE}=$ CRD Ejected.

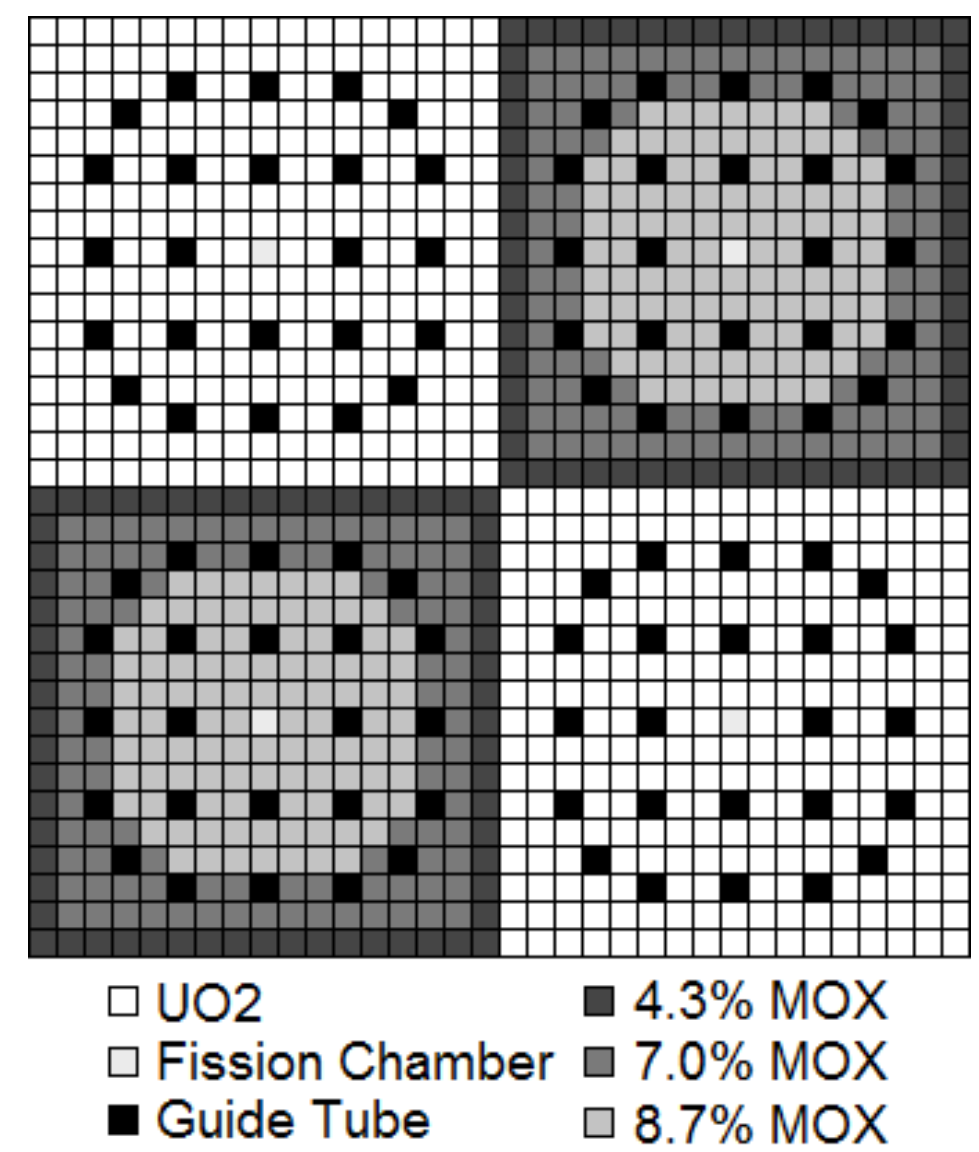

Figure 14. C5G7 southeast quadrant assembly geometry [18] 


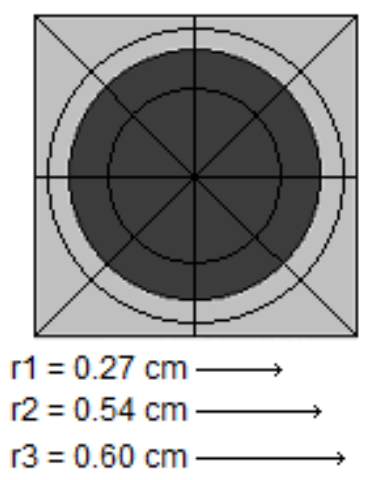

Figure 15. C5G7 pin cell discretization with corresponding radii.
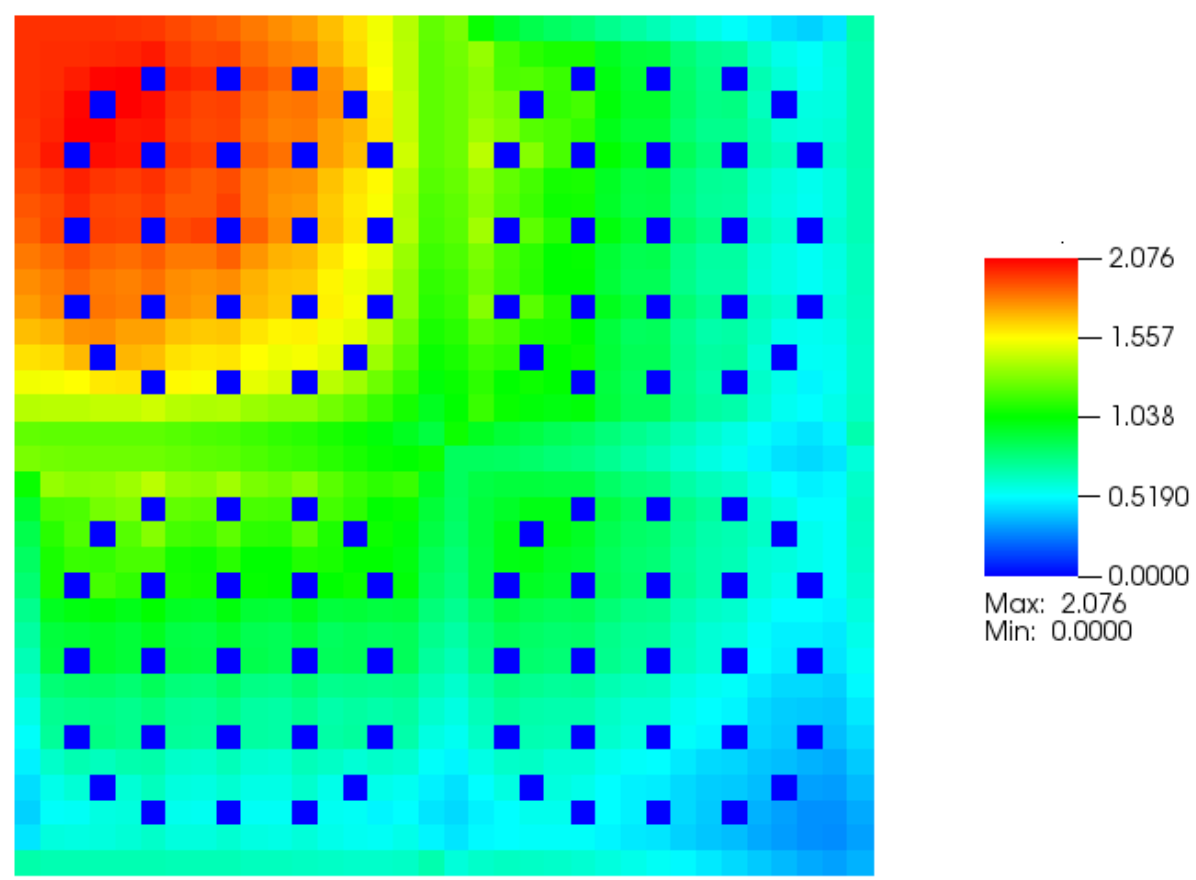

Figure 16. Steady-state relative pin cell power distribution for C5G7 southeast quadrant 


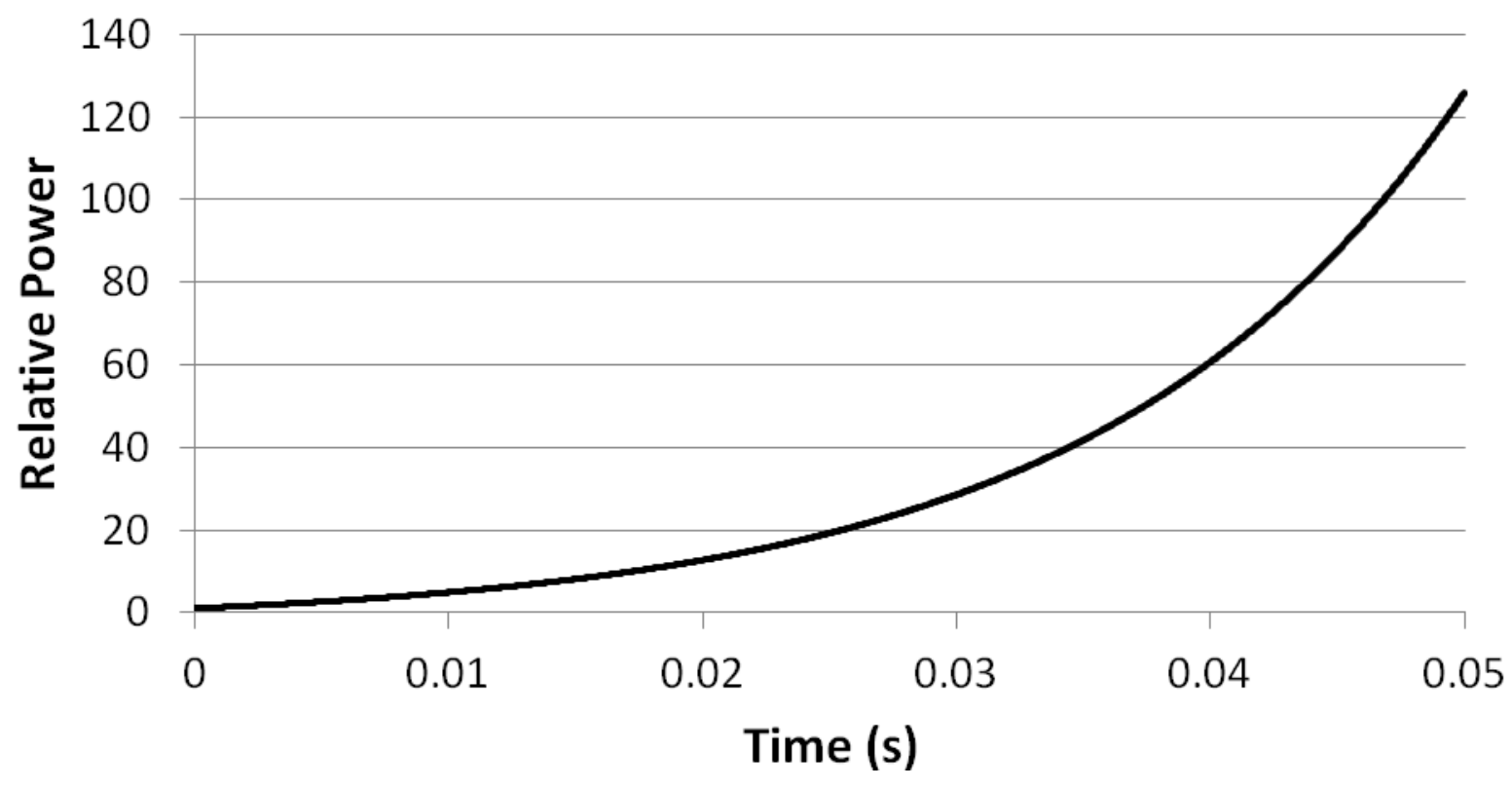

Figure 17. Relative core power for reference solution for C5G7

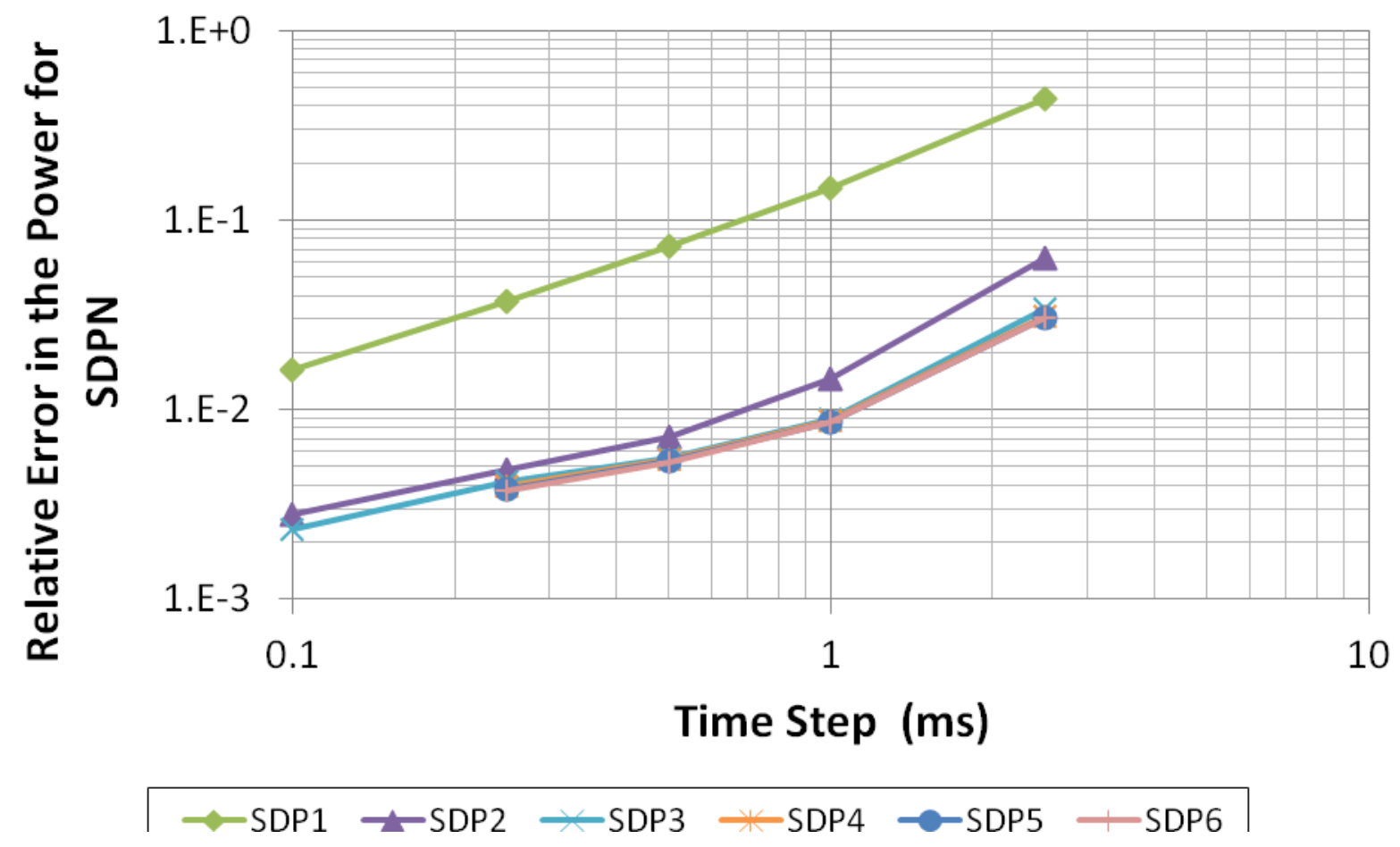

Figure 18. Relative error in final power for SDP1-6 as a function of time step 


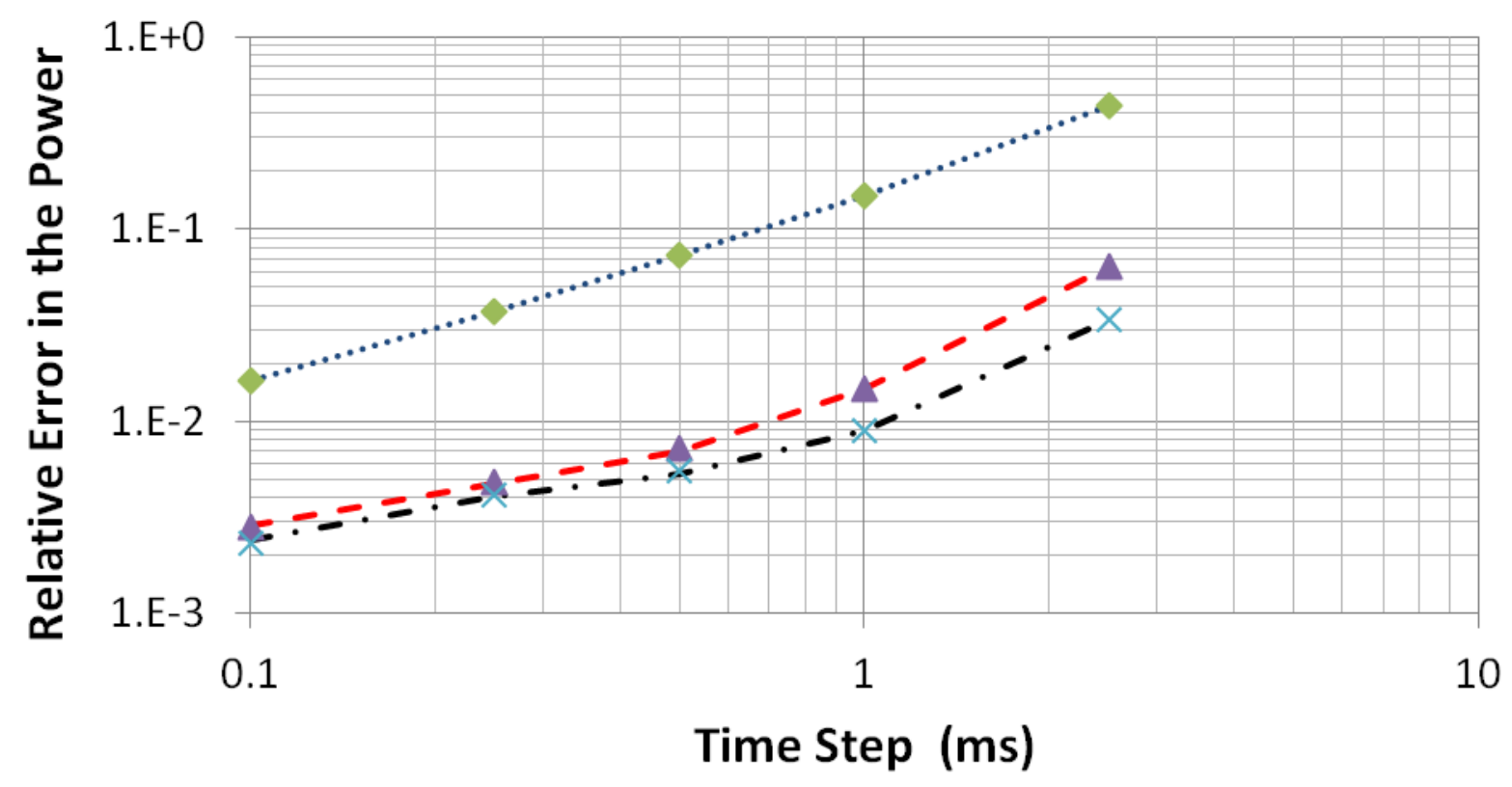

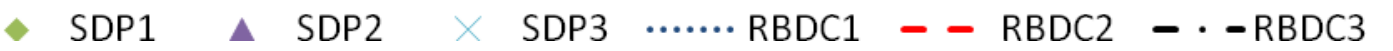

Figure 19. Relative error in the final power for SDP1-3 and RBDC1-3, illustrating that the SDP solutions closely track the corresponding RBDC solutions

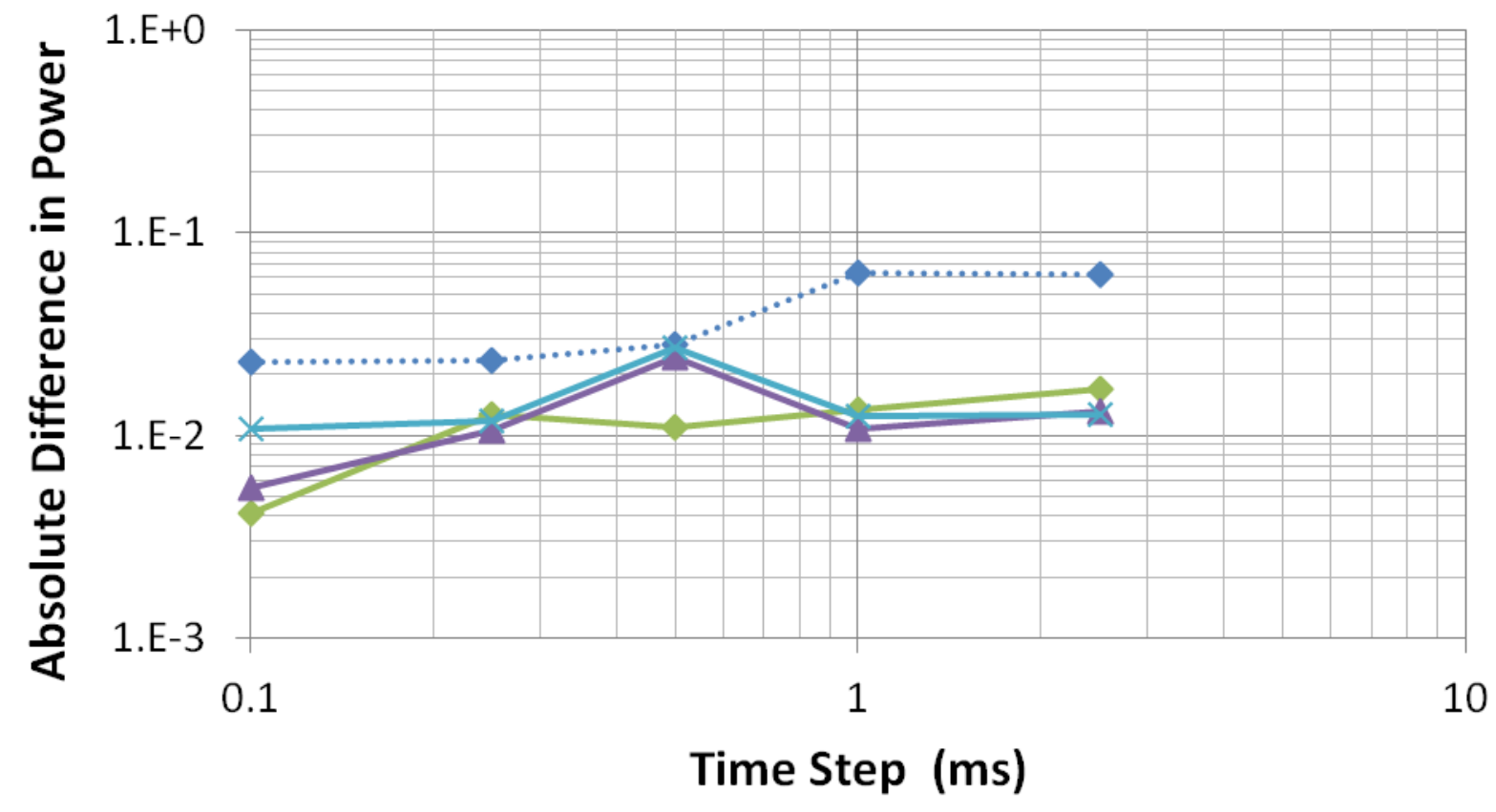

$\ldots \leftrightarrow$ IBDC1 v RBDC1 $\longleftarrow$ SDP1 v RBDC1 —SDP2 v RBDC2 $\longleftarrow$ SDP3 v RBDC3

Figure 20. Absolute difference in the final power 

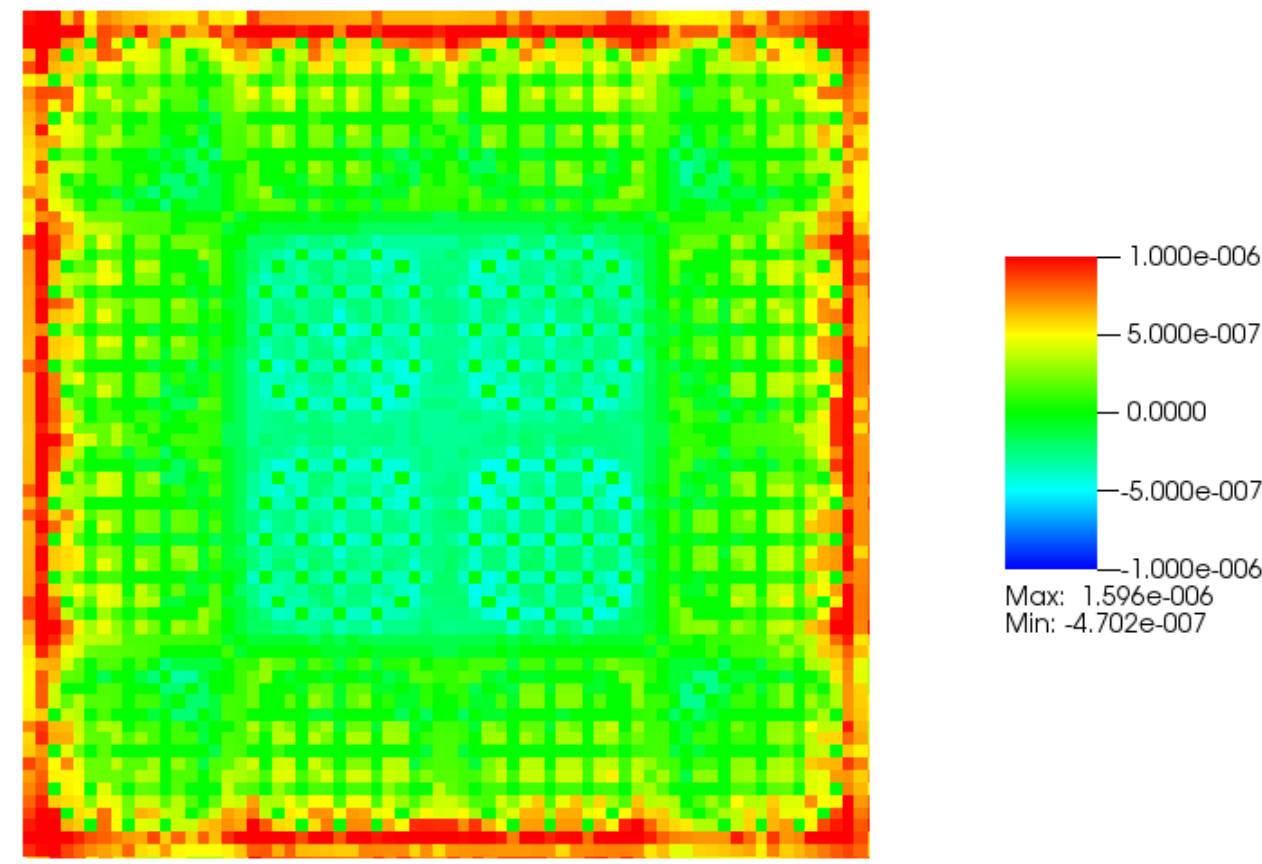

Max: $1.596 \mathrm{e}-006$

Min: $-4.702 \mathrm{e}-007$

Figure 21. Relative error in the relative pin power for ISDP1 at $0.05 \mathrm{~s}$
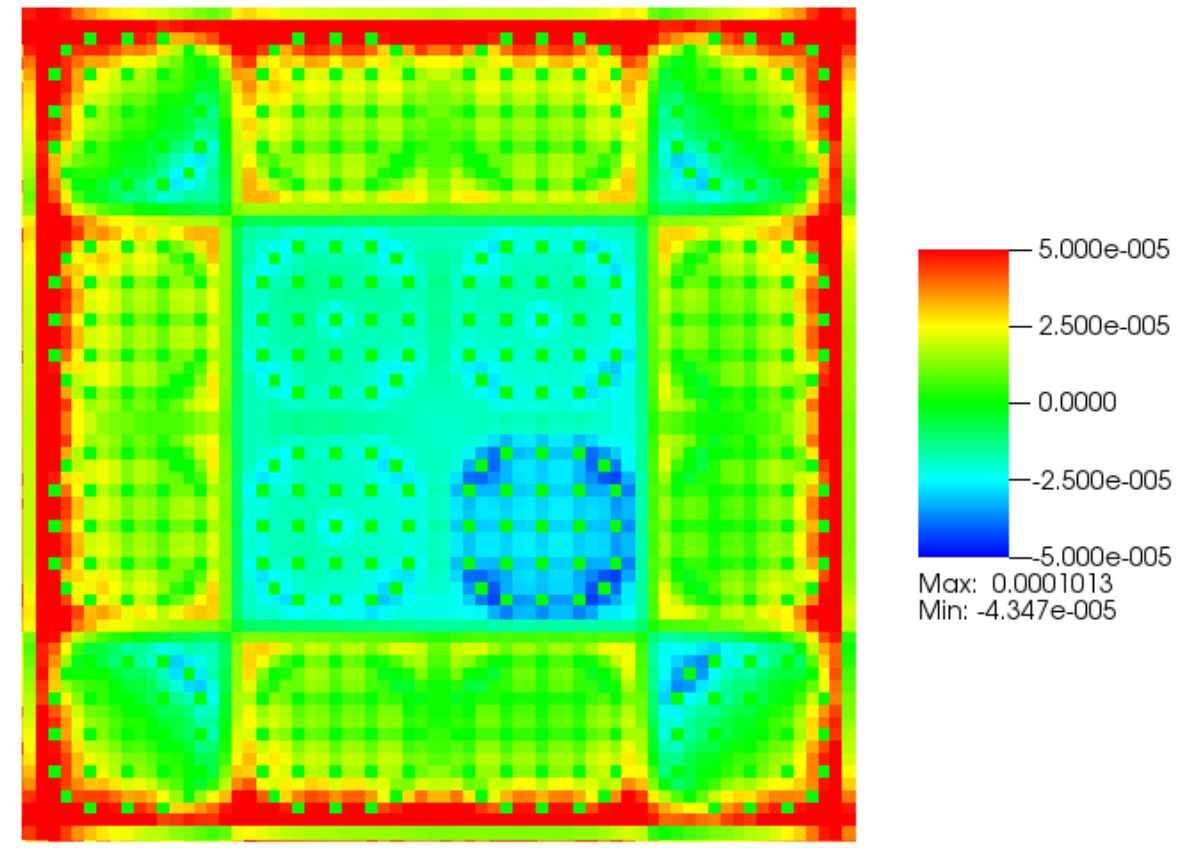

Max: 0.0001013

Min: $-4.347 \mathrm{e}-005$

Figure 22. Relative error in the relative pin power for IBDC1 at $0.05 \mathrm{~s}$ 